\title{
Planar distribution of the galaxies in the Local Group: a statistical and dynamical analysis $\star$
}

\author{
S. Pasetto and C. Chiosi \\ Department of Astronomy, Padova University, Vicolo dell'Osservatorio 2, 35122, Padova, Italy \\ e-mail: [pasetto; chiosi]@pd.astro.it
}

Received 13 July 2006 / Accepted 17 November 2006

ABSTRACT

\begin{abstract}
Aims. Basing on the projected distribution of the galaxies in the Local Group, Sawa \& Fujimoto found that they all seem to distribute on a rather thin plane containing the two mayor local galaxies of the Local Group, Milky Way and Andromeda, and all their dwarf satellites. As their conclusion could be severely biased by projectional distortion effects, we re-analyse the whole issue using a different approach. In brief, adopting known data on positions and distances, we make use of the analytical geometry and look for the plane that minimizes the distances of all galaxies to it. A planar distribution is indeed found that, however, does not coincide with the plane found by Sawa \& Fujimoto. Why? The second part of this study is devoted to answer this question and to find a dynamical justification for the planar distribution.

Methods. To this aim, we apply the Hamilton Method (Minimum Action) to investigate the dynamics of the two major system of the Local Group, Milky Way and Andromeda, under the action of external forces exerted by nearby galaxies or groups external to the Local Group.

Results. We find that the planar distribution is fully compatible with the minimum action and that the external force field is likely parallel to the plane. It pulls the galaxies of the Local Group without altering their planar distribution. Special care is paid to evaluate the robustness of this result.

Conclusions. In this paper we have examined the spatial distribution of galaxies in the Local Group. They are confined to a plane that can be statistically and dynamically understood as the result of the Minimum Action. The planar distribution seems to be stable for a large fraction of the Hubble time. The external force field, that has likely been constant over the same time interval, does not alter the planar distribution as it is nearly parallel to it. Effects due to undetected halos of sole Dark Matter are briefly discussed. They could be a point of uncertainty of the present study.
\end{abstract}

Key words. stellar dynamics - Local Group - galaxies: dwarf

\section{Introduction}

Recent reviews of the state-of-art of our understanding of the structure, past history, galaxy content, and stellar populations in individual galaxies of the Local Group (LG) are by van den Bergh (1999), Mateo (1998) and Grebel (2001). However, our present knowledge of the proper motions of Andromeda (M 31) and most galaxies of the LG is dramatically insufficient and inadequate to correctly reconstruct the past and present structure of the LG. As a matter of facts, no orbital parameters can be derived with adequate precision to constrain the space of velocities for all known objects. As a consequence of it, the dynamical evolution of the LG galaxies, and the dynamical origin of the dwarf satellites orbiting around and interacting with the two major galaxies, i.e. Milky Way (MW) and M 31, are not clear.

Over the years, many authors addressed these issues both observationally and theoretically, with the aim of understanding the evolution of the LG galaxies. Since the first observations of Barnard (1917), perhaps the most important steps toward understanding the nature of the LG is the determination of the proper motion and tangential motion of M 31, which unfortunately are still poorly known. The first important study dealing with the evolution of the LG, supposedly in isolation, is by

* Appendix A is only available in elctronic form at http://www . aanda. org
Kahn \& Woltjer (1959). The authors based their study on the spherical gravitational collapse and expansion approximation. As consequence of it, with the aid of the third Kepler's law they predicted only radial orbits for the MW and M 31 from the origins to the present time. The possibility of a tangential motion for M 31 with respect to the MW was automatically ruled out. Another model of the LG based on pure radial orbits for M 31 and MW has been presented by Lynden-Bell \& Lin (1977). The authors made use of the second law of Newton applied to the motion of the two major components of the LG. Yahil et al. (1977) focused on the determination of the circular velocity of our own galaxy using the LG as reference system. They studied the origin of the LG angular momentum and for the first time emphasised how the tidal force exerted by external proto-groups could be the cause of the origin of the LG angular momentum, and how this interpretation should be preferred to the cosmological origin proposed by Hoyle (1951), Peebles (1969) and Thuan $\&$ Gott (1977). They supposed that the spin-orbit coupling in the first stages of the Universe could occur, even if the angular moment gained by the spin of Dark Matter halos and then transferred to the orbits could be too small even for the largest eccentricity orbits (Ostriker \& Peebles 1973; Ostriker et al. 1974). As these conclusions were not in agreement with previous studies, they spurred the first attempts to measure the proper motions of M 31 (different from zero). 
Gott \& Thuan (1978) first presented the equations governing the motion of the LG members. This study, however, still made use the radial approximation for the relative motion of MW and M 31. In their picture, thanks to the Thompson scattering before the recombination time $(z \approx 1500)$, the matter is coupled with radiation and it does not present random motions but only the motion of the Hubble flow. After recombination, the matter decouples from radiation: therefore galaxies and galaxy clusters can freely form thanks to the small unstable fluctuations of the density (Peebles 1974; Gunn \& Gott 1972). In this context, the equations for the motion of MW and M 31 have been written for the first time. This yielded an upper limit for the redshift at which the proper motions originated, $z<1500$. At earlier epochs, speaking of proper motions was not physically meaningful. These conclusions are, however, based on the fact that the vorticity of the CMB as well as that of the initial conditions are null. Nowadays minor anisotropies of the CMB have been discovered by WMAP (Bennett et al. 2003; Saha et al. 2006) thus somewhat weakening the above assumptions. Notwithstanding the new observational and theoretical developments, the study of Gott \& Thuan (1978) is still a landmark on the origin of the LG angular momentum.

Subsequently, Lynden-Bell (1981) presented the equations for a non purely radial motion of M 31. The history of the orbital equations for the LG galaxies became even more complicated with results intermediate to those by Lynden-Bell (1981) and Einasto \& Lynden-Bell (1982).

An independent treatment of the equations of motion for the LG members was presented by Mishra (1985). Using the solutions of the restricted 3-body problem and assuming the ellipticity of $\epsilon \approx 0.9$ estimated by Einasto \& Lynden-Bell (1982) and Yahil et al. (1977), it was argued that M 31 and MW are on a linear orbit and that the minor galaxies maintain a planar motion throughout at all times.

A milestone along the road of the evolution and formation of the LG was set by the Nearby Galaxies Catalog (hereinafter NBG) of Tully (1988), a sample of 2367 galaxies with systematic velocities less than $3000 \mathrm{~km} \mathrm{~s}^{-1}$. The spatial location of the NBG galaxies is shown in Fig. 1 to give an idea of structure of the Local Universe. A first systematic investigation of the effect of the external galaxies on the LG has been presented in Raychaudhury \& Lynden-Bell (1989). Studying the influence of the gravitational quadrupole interaction by external galaxies on the LG, the authors proved that the LG is not tidally isolated and presented a new set of equations for the motion of its galaxies. Even if the equations and the proper motion of M 31 given by Raychaudhury \& Lynden-Bell (1989) are not fully correct (e.g. see Dunn \& Laflamme 1993), the fundamental result still remains: the $L G$ is not isolated and the proper motion of $M 31$ is not null.

In the same years, a flourish of theoretical studies began (Peebles 1989, 1994, 1995). In Peebles (1989) the equations of motion were revised with the aid of the Hamiltonian Principle and a new method for tracing back the orbits of the LG galaxies was presented and subsequently adapted to the LG problem by Peebles et al. (1989) and Peebles (1994), together with new determinations of the tangential motion of M 31 (Peebles 1990, 1995). In the series of studies by Peebles and collaborators, and other independent groups as well, an interesting result was obtained (Shaya et al. 1995; Peebles 1995; Sharpe et al. 1999; Peebles et al. 2001; Goldberg 2001b): the solutions for the local minimum or stationary points of the action are compatible with a tangential motion of M 31 as large as the radial motion.

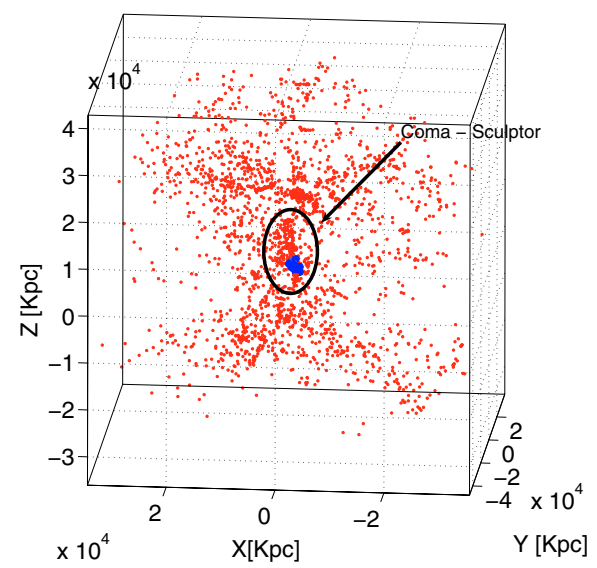

Fig. 1. A sketch of the spatial distribution of the NBG galaxies in the Local Universe. The Sculptor-Coma cluster is highlighted and bigger dots are used for M 31, MW and the LG galaxies of 1. From Tully \& Fisher (1988).

Following the same methodology, Dunn \& Laflamme (1993) independently derived the value for the proper motion of M 31 $\left(\mu_{l}, \mu_{b}\right)=(0.126,0.0048) \mathrm{mas} / \mathrm{yr}$. Despite this great improvement in the knowledge of the LG, new problems arose regarding its cosmological origins as noted by Governato et al. (1997). They pointed out the strong dependence of the theoretical models on the cosmological parameters for the peculiar velocities of galaxies in CDM context. The same dependence has been suspected for some observational constraints, e.g. for the distances or the radial velocities (Valtonen et al. 1993; Byrd et al. 1994). Recently a new determination of the mass of M 31 has been derived by Evans \& Wilkinson (2000). In contrast with all previous dynamical estimates, the mass assigned to M 31 is smaller than the mass of MW. This result can be explained recalling that it stems from the notion that the velocity vector of M 31 is purely radial and that the mass is derived from velocity arguments. One may argue that the lower mass assigned to M 31 could be taken as an hint that the motion of M 31 is not merely radial.

Moreover, attempts were made to find the spatial distribution of the LG galaxies, e.g. Hartwick (2000) suggested that the galaxies crowd on a highly flattened ellipsoid with axial ratios $(a, b, c)=(1.00,0.51,0.19)$ which to a good approximation can be considered as a planar distribution. Another problem under investigation was the anisotropic distribution of inner sub-haloes with respect to larger haloes in relation to the Holmberg effect (Sales \& Lambas 2004). General consensus on this issue has not yet been reached (Knebe et al. 2004). In particular it is unclear and matter of debate whether disruption-tidal effects can create the apparent polar alignment of the dwarf satellites around the mother galaxy or, for the particular case of the LG, the position of the dwarf galaxies is the consequence of peculiar directions of pre-existing cosmological filaments. See for instance Fig. 1 which displays the spatial distribution of the NBG galaxies. In a recent paper, Kroupa et al. (2005) suggest a planar distribution of the satellites of MW, which however could also be explained as a consequence of the distribution of sub-haloes (Zentner et al. 2005) in the early cosmological stages (Kang et al. 2005). Another important result for the sub-halo alignment in cosmological simulations was obtained by Libeskind et al. (2005). The same problem is under investigation for M 31 (Koch \& Grebel 2006). Finally, Sawa \& Fujimoto (2005) have recently proposed a new dynamical model based on the existence an orbital plane for M 31 and MW. 


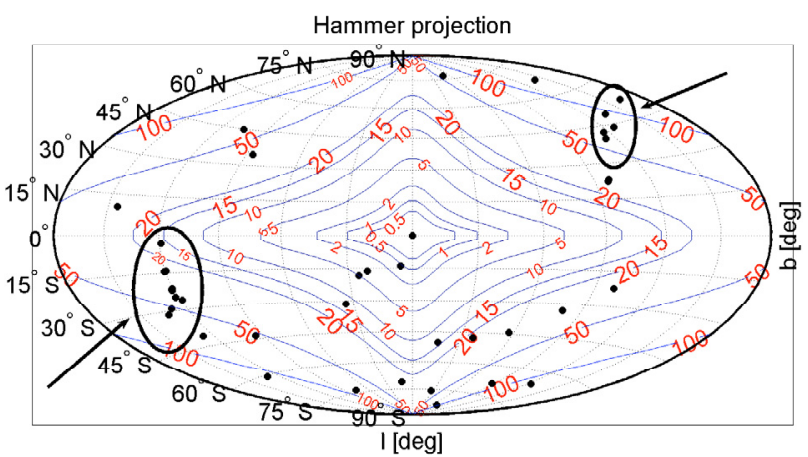

Fig. 2. Hammer's projection with the amplitude of distortion (in percent) according to the code $1=$ no distortion, $100=$ extremely high distortion. The heavy dots are the galaxies of Table 1 . The arrows indicate the regions of larger concentration of objects that could be interpreted as hints of underlying 3D structures. They, however, occur in regions of large distortion.

In this paper we address some of the above issues. The plan is as follows. In Sect. 2 we examine the spatial distribution of the LG galaxies: starting from the analysis of Sawa \& Fujimoto (2005) and using a different procedure (Principal Component Analysis) we find that a common plane really exists but different from the one found by Sawa \& Fujimoto (2005). We then proceed to study the dynamics of the LG. In Sect. 3 we present the Hamilton method of Constrained Minimum Action. In Sect. 4 we present the samples of galaxies in the Local Universe we have adopted to study the dynamics of the LG and the effects on this by nearby galaxies. In Sect. 5 we minimize the Action and present the results. In Sect. 6 we examine in detail the robustness of the procedure we have adopted. In Sect. 7 we evaluate the effect of external galaxies on the dynamics of the LG. The main result is that the orbits of the dominant galaxies of the LG are dynamically consistent with a plane that within the uncertainties coincides with the one derived in Sect. 2 from mere geometrical considerations. This is indeed the plane that minimizes the action. Several important implications of this finding are discussed in the above sections. Finally, in Sect. 8 we draw some concluding remarks.

\section{Is there a plane on which LG galaxies crowd?}

As already mentioned, from the visual inspection of the skyprojected spatial distribution of the LG galaxies Sawa \& Fujimoto (2005) suggest the existence of an orbital plane for MW, M 31 and satellites. However the idea is not new. 2D studies have already been employed to establish planes in the LG. Limiting ourselves to a few examples, Hartwick (2000) suggested a flat ellipsoid which is not too different from a plane; studies of the LG dynamics, see for instance Kahn \& Woltjer (1959) and Raychaudhury \& Lynden-Bell (1989). Finally Kunkel (1979), Grebel et al. (1999) and Fusi Pecci et al. (1995) suggested that the satellite dwarf galaxies of the MW and M 31 lie on planes.

We suspect, however, that the plane found by Sawa \& Fujimoto (2005) largely results from distortion effects affecting the visual inspection of spatial structures. Therefore, in this section we reconsider the whole subject and check whether such a plane can be derived from other objective methods.

Our analysis of the galaxies distribution stands on the Mateo (1998) sample of LG galaxies (see the entries of Table 1). Whenever required the radial velocities are taken from

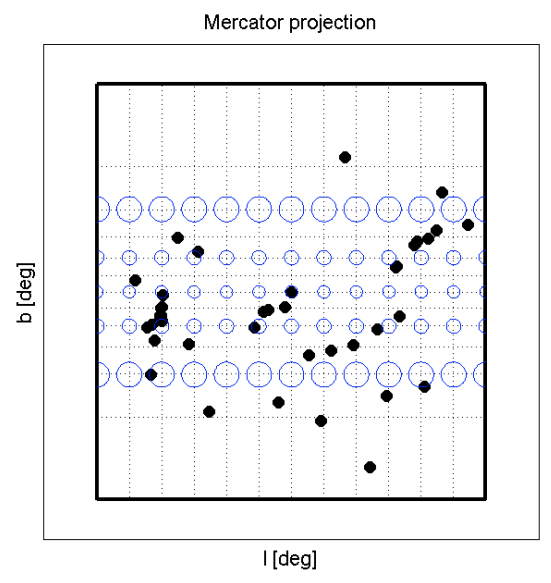

Fig. 3. Mercator's projection with Tissot's circles. The Mercator projection provides a good representation near the equatorial plane and a very poor one towards the poles. The lack of galaxies near the galactic plane is due to Galactic absorption. Clearly the apparent distribution is not uniform. There seem to be several 2D structures that could perhaps hint for real 3D structures. However, the same remark made in Fig. 2 would apply also here.

Tully \& Fisher (1988), however updated to more recent determinations if available (see below). The same sample has been used by Sawa \& Fujimoto (2005).

Following Sawa \& Fujimoto (2005) we start by looking at the projected distribution of the LG members on the celestial sphere. It is soon evident that this way of proceeding is severely biased by the type of projection in use. To illustrate the point, in Fig. 2 we show the Hammer projection on which the contours of projection-induced distortion on the map are superposed. The distortion is measured by the ratio of the infinitesimal areas given by the jacobian of the transformation matrix between the cartesian coordinate system and the projected coordinate system. The amplitude of distortion is given in percent, i.e. normalised to an un-distorted projection. As a matter of fact the major clustering of objects that could suggest peculiar spatial structures such as filaments, planes etc, actually occurs in regions of the Hammer plane with distortion amounting to about 50-75 percent. Therefore, visual inspections of the projected distribution to infer spatial structures could lead to wrong conclusions. Other kinds of projection do not make a better job. For instance in Figs. 3 and 4 we show the Mercator and Cassini projections, respectively. We have added the Tissot's circles, which are equal circles on the celestial sphere to better visualise the effects of projectional distortions. In principle, the combination of these two projections could improve upon the correct view of different areas on the celestial sphere. In the Mercator projection all the lines with the same angles with respect to the meridians are straight lines, and thus reduce the distortion near the equatorial plane of the $\mathrm{MW}\left(l<86^{\circ}\right.$ is conventionally adopted). Unfortunately, as all dwarf galaxies of the LG have $|b|<84^{\circ}$, in the direction $(l, b) \approx(0,0)$ they are masked by the Galactic Plane, even the Mercator projection does not help us very much. The Cassini projection ensures instead a good approximation toward the Galactic Pole. Once again the few apparent structures that could hint for real 3D structures are located near regions of large distortion. Therefore, the main result of this first analysis is that the 3D structure of the LG when projected onto 2D surfaces could mimic planar distributions that may not exist in reality.

For the above reasons, we decided to approach the problem in a different way, i.e. making use of the analytical geometry 
Table 1. Parameters of LG galaxies used in this study.

\begin{tabular}{|c|c|c|c|c|c|c|c|c|}
\hline Galaxy Name & Other name & $l$ & $b$ & $r$ & Group & $x$ & $y$ & $z$ \\
\hline WLM & DDO 221 & 75.9 & -73.6 & 925 & LGC & -55.1 & 253.3 & -887.4 \\
\hline NGC55 & & 332.7 & -75.7 & 1480 & LGC & -316.3 & -167.7 & -1434.1 \\
\hline IC10 & UGC192 & 119 & -3.3 & 825 & M 31 & 407.8 & 720.4 & -47.5 \\
\hline NGC147 & DDO3 & 119.8 & -14.3 & 725 & M 31 & 357.6 & 609.6 & -179.1 \\
\hline And III & & 119.3 & -26.2 & 760 & M 31 & 342.2 & 594.7 & -335.5 \\
\hline NGC185 & UGC396 & 120.8 & -14.5 & 620 & M 31 & 315.9 & 515.6 & -155.2 \\
\hline NGC205 & M110 & 120.7 & -21.1 & 815 & M 31 & 396.7 & 653.8 & -293.4 \\
\hline M32 & NGC221 & 121.2 & -22 & 805 & M 31 & 395.1 & 638.4 & -301.6 \\
\hline M 31 & NGC224 & 121.2 & -21.6 & 770 & M 31 & 379.4 & 612.4 & -283.5 \\
\hline And I & & 121.7 & -24.9 & 805 & M 31 & 392.2 & 621.2 & -338.9 \\
\hline SMC & NGC292 & 302.8 & -44.3 & 58 & MW & -14.0 & -34.9 & -40.5 \\
\hline Sculptor & & 287.5 & -83.2 & 79 & MW & 5.7 & -8.9 & -78.4 \\
\hline LGS3 & Pisces & 126.8 & -40.9 & 810 & M 31 & 375.2 & 490.2 & -530.3 \\
\hline IC1613 & DDO8 & 129.8 & -60.6 & 700 & M 31/LGC & 228.5 & 264.0 & -609.8 \\
\hline And II & & 128.9 & -29.2 & 525 & M 31 & 296.3 & 356.7 & -256.1 \\
\hline M33 & NGC598 & 133.6 & -31.3 & 840 & M 31 & 503.5 & 519.8 & -436.4 \\
\hline Phoenix & & 272.2 & -68.9 & 445 & MW/LGC & 2.4 & -160.1 & -415.2 \\
\hline Fornax & & 237.1 & -65.7 & 138 & MW & 39.3 & -47.7 & -125.8 \\
\hline EGB0427+63 & UGCA92 & 144.7 & 10.5 & 1300 & M 31 & 1051.7 & 738.6 & 236.9 \\
\hline LMC & & 280.5 & -32.9 & 49 & MW & 1.0 & -40.5 & -26.6 \\
\hline Carina & & 260.1 & -22.2 & 101 & MW & 24.6 & -92.1 & -38.2 \\
\hline Leo A & DDO69 & 196.9 & 52.4 & 690 & MW/N3109 & 411.3 & -122.4 & 546.7 \\
\hline Sextans B & DDO70 & 233.2 & 43.8 & 1345 & N3109 & 590.0 & -777.3 & 930.9 \\
\hline NGC3109 & DDO236 & 262.1 & 23.1 & 1250 & N3109 & 166.5 & -1138.9 & 490.4 \\
\hline Antlia & & 263.1 & 22.3 & 1235 & N3109 & 145.8 & -1134.4 & 468.6 \\
\hline Leo I & & 226 & 49.1 & 250 & MW & 122.2 & -117.7 & 189.0 \\
\hline Sextans A & DDO75 & 246.2 & 39.9 & 1440 & N3109 & 454.3 & -1010.8 & 923.7 \\
\hline Sextans & & 243.5 & 42.3 & 86 & MW & 36.9 & -56.9 & 57.9 \\
\hline Leo II & DDO93 & 220.2 & 67.2 & 205 & MW & 69.2 & -51.3 & 189.0 \\
\hline GR8 & DDO155 & 310.7 & 77 & 1590 & GR8 & -224.7 & -271.2 & 1549.2 \\
\hline Ursa Minor & DDO199 & 105 & 44.8 & 66 & MW & 20.6 & 45.2 & 46.5 \\
\hline Draco & DDO208 & 86.4 & 34.7 & 82 & MW & 4.3 & 67.3 & 46.7 \\
\hline Sagittarius & & 5.6 & -14.1 & 24 & MW & -14.7 & 2.3 & -5.8 \\
\hline SagDIG & UKS1927-177 & 21.1 & -16.3 & 1060 & LGC & -940.7 & 366.3 & -297.5 \\
\hline NGC6822 & DDO209 & 25.3 & -18.4 & 490 & LGC & -411.9 & 198.7 & -154.7 \\
\hline DDO 210 & Aquanus & 34 & -31.3 & 800 & LGC & -558.2 & 382.2 & -415.6 \\
\hline IC5152 & & 343.9 & -50.2 & 1590 & LGC & -969.4 & -282.2 & -1221.6 \\
\hline Tucana & & 322.9 & -47.4 & 880 & LGC & -466.6 & -359.3 & -647.8 \\
\hline UKS2323-326 & UGCA 438 & 11.9 & -70.9 & 1320 & LGC & -414.1 & 89.1 & -1247.3 \\
\hline Pegasus & DDO216 & 94.8 & -43.5 & 955 & LGC & 66.5 & 690.3 & -657.4 \\
\hline
\end{tabular}

The coordinate system is centered on the MW. The $X$ axis points from the centre of MW to the Sun, located at $R_{\odot}=8.5 \mathrm{kpc}$, the $Z$ axis points to the North Galactic Pole, and the third axis forms a right handed coordinate system. Distances are in kpc. The same sample has been used by Sawa \& Fujimoto (2005). We do not show the radial velocities because they are not used in this paper.

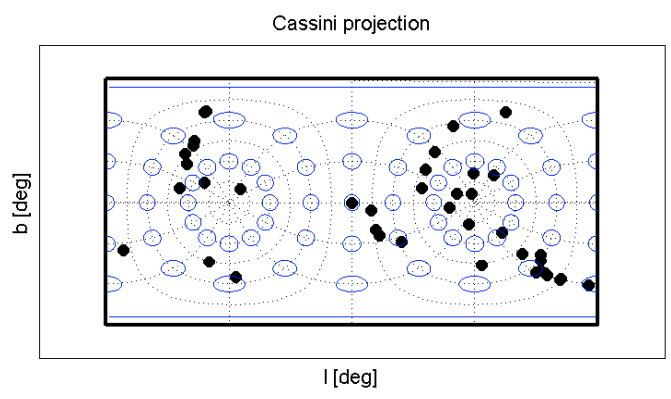

Fig. 4. Cassini's projection with Tissot's circles. The North and South Galactic Poles are now well represented. Once again a few 2D structures can be noticed. They, however are located in regions of high distortion so that the same remark made in Fig. 2 would apply also here

to investigate the idea of Sawa \& Fujimoto (2005). We start by assuming that the Sawa \& Fujimoto (2005) conclusion is true, i.e. LG galaxies crowd on a plane, but check it. The method we have adopted is ultimately the Principal Components Analysis (see for instance Jobson 1994). Given $N$ galaxies (of the LG), with coordinates $\boldsymbol{x}^{(g)}, g=1 \ldots N$ we want to find the plane that best approximates their spatial distribution. In other words, given $N$ points, which are the principal directions of the plane best approximating these points? aims

The Hessian normal representation of a plane is suited to our

$\hat{\boldsymbol{n}} \cdot \boldsymbol{x}=-c$

where the $3 \mathrm{D}$ vector $\hat{\boldsymbol{n}}$ is the unitary vector orthogonal to the plane, and $c$ the distance from the origin of the coordinate system in use. The reason for choosing this geometrical representation of the plane instead of the classical one $a x+b y+c z+d=0$ is that in the Hessian formalism the distance of any given point to the plane $\tilde{\boldsymbol{x}}$ is particularly simple:

$D_{i}=\hat{\boldsymbol{n}} \cdot \tilde{\boldsymbol{x}}_{i}+c$ 

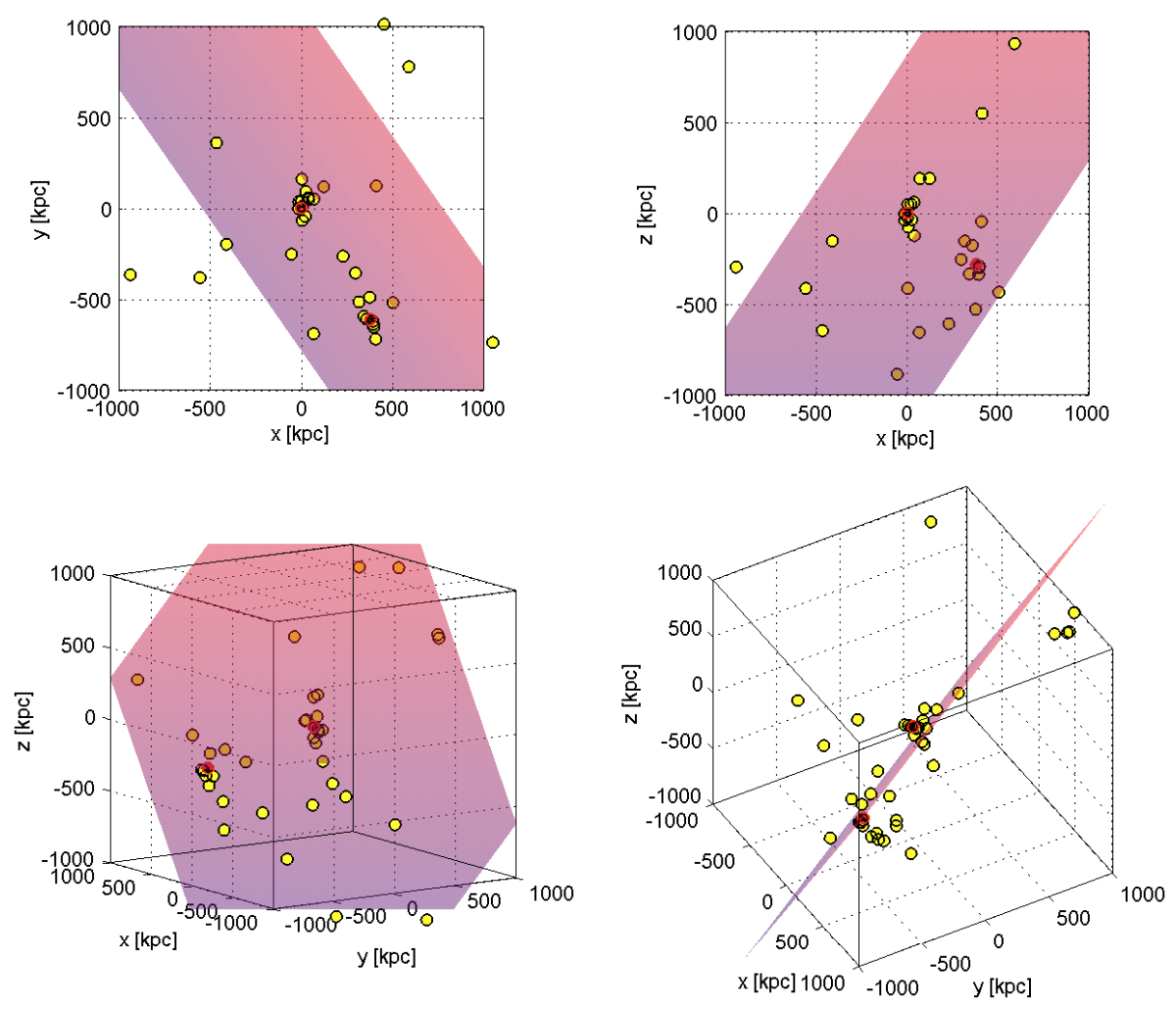

Fig. 5. Projection onto the coordinate planes $(X, Y)$ and $(X, Z)$ as indicated, and two 3D views (bottom left and right panels) of the our plane best minimizing the distance (location) of the LG galaxies. The $(X, Y)$ projection (top left panel) is parallel to the equatorial plane of the MW. The big red filled circles are MW and M 31. The yellow circles are the dwarf satellites on either sides of the plane. Looking at the 3D view in which the plane is seen edge-on (bottom right), MW and M 31 are very close to it and within the uncertainty can be considered as belonging to it. See the text for details.

Then we minimize the equation for the distance $D$ for all points

$D=\sum_{g=1}^{N}\left|\boldsymbol{n} \cdot \boldsymbol{x}^{(g)}+c\right|^{2}$.

The simplest way is given by the Lagrange multipliers. First we introduce the condition ensuring the ortho-normality of the vector $\hat{\boldsymbol{n}}$

$|\boldsymbol{n}|^{2}-1=0$

and get the system of equations

$$
\left\{\begin{array}{l}
\sum_{g=1}^{N}\left[\left(\boldsymbol{n} \cdot \boldsymbol{x}^{(g)}+c\right) x_{i}^{(g)}\right]=\lambda n_{i} \forall i=1 \ldots 3 \\
\sum_{g=1}^{N}\left(\boldsymbol{n} \cdot \boldsymbol{x}^{(g)}+c\right)=0 \\
|\boldsymbol{n}|^{2}-1=0
\end{array}\right.
$$

(see e.g. De Marco 1994). It follows that distances are linked to the eigen-values of the eigen-system $\boldsymbol{A} \cdot \boldsymbol{n}=\lambda \boldsymbol{n}$ that can be derived from the system (5), where the matrix $\boldsymbol{A}$ is given by

$\boldsymbol{A}_{i j} \equiv \sum_{g=1}^{N}\left(\left(x_{j}^{(g)}-\frac{1}{N} \sum_{h=1}^{N} x_{j}^{(h)}\right) x_{i}^{(g)}\right)$.

The resulting eigen-values, corresponding to 3 values for the minimum of $D$, are

$$
\begin{aligned}
& D^{(1)}=\lambda^{(1)}=2.01 \times 10^{7} \\
& D^{(2)}=\lambda^{(2)}=1.05 \times 10^{7} \\
& D^{(3)}=\lambda^{(3)}=3.88 \times 10^{6},
\end{aligned}
$$

which yield the eigen-vectors:

$$
\begin{aligned}
& \boldsymbol{n}^{(1)}=(0.19,-0.51,0.83) \\
& \boldsymbol{n}^{(2)}=(0.66,0.69,0.27) \\
& \boldsymbol{n}^{(3)}=(0.72,-0.50,-0.47) .
\end{aligned}
$$

Each of these vectors corresponds to a plane. Therefore we have 3 orthogonal planes out of which we have to select the one yielding the absolute minimum distances. The planes are referred to an orthogonal reference system co-aligned with the MW located at the origin. Clearly the plane to choose is the third one. Finally, for the sake of the comparison with other studies, it is worth noting here that the normal vector of this plane is along the direction $(l, b)=\left(-136^{\circ},-28^{\circ}\right)$.

The situation of the plane is shown in Fig. 5 where the top left and right panels show the LG galaxies projected onto the plane of the MW and perpendicular to it, whereas the bottom left and right panels show the same galaxies projected face-on and edge-on onto the plane minimizing $D$. From the lower right panel of Fig. 5 we see that the two dominant galaxies (MW and M 31) do not strictly belong to the plane. However, considering the uncertainties in the position of each galaxy, we may reasonably conclude that also the two major galaxies of the system belong to the plane ${ }^{1}$.

Starting from this first result we may also assume that the geometrical plane contains the barycentre of the system. This in not true in general but limited to the LG. It follows that the distribution of the baryonic (and dark) matter is symmetric with respect to this plane. Furthermore, the fact that the two most massive galaxies belong to the plane could perhaps indicate that the effect, if any, of still undetected massive bodies influencing the LG dynamics is small and likely was such in past. On the problem of the mass tracers (Davis \& Peebles 1983; White et al. 1988) we will come back in Sect. 3. To conclude we may provisionally assume that

1. No pure dark bodies should exist that could significantly affect the dynamics of the LG (see Sect. 3 for more details). Therefore, the dominant galaxies (with the largest mass) determining the motion of the dwarf satellites are MW and

\footnotetext{
${ }^{1}$ A detailed astrophysical explanation (not geometrical) for the errors in the definition of the plane will be given later in this section.
} 


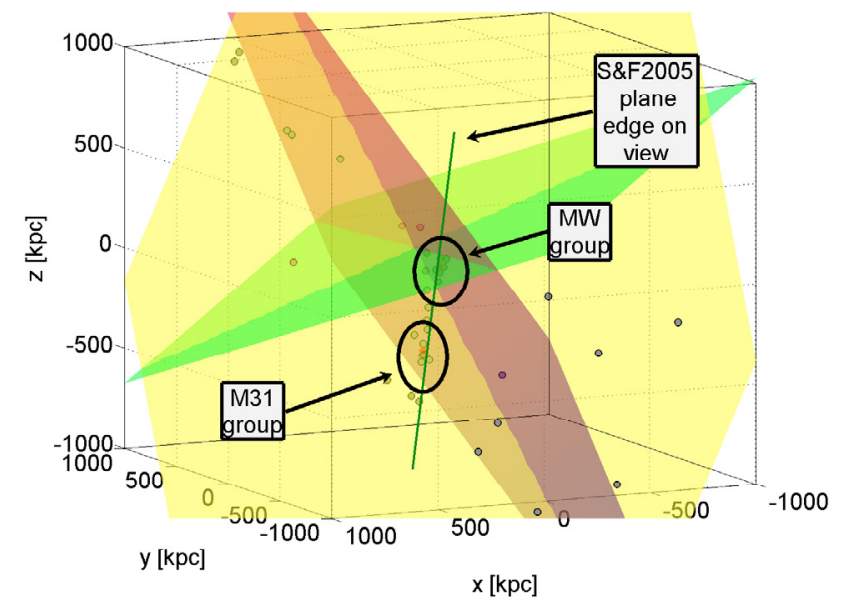

Fig. 6. The 3D view of the three planes we have found with Eq. (7) together with the plane found by Sawa \& Fujimoto (2005) seen edge-on and the galaxies of the LG. The two encircled zones highlight the position of MW and M 31 and their satellites. None of our planes coincides with that of Sawa \& Fujimoto (2005). See the text for more details.

M 31. In other words, in the CDM hierarchical accretion scenario, if there were a pure dark body as massive as the MW or M 31 halos, we would expect the dwarf galaxies (which owing to their small mass are good traces of the global gravitational potential) not to lie on the same plane containing MW and M 31.

2. The problem of the small number of known dwarf galaxies, (see e.g. Klypin et al. 1999; Moore et al. 1999), as compared to the expected one is not relevant for the dynamics of the LG, see e.g. Mateo (1998) or van den Bergh (1999). In other words, even in presence of a large number of dwarf satellites, less massive that about $10^{7} M_{\odot}$, the dynamics of the known LG dwarf satellites is not strongly affected. There is indeed no reason for a group of about 100 unseen satellites to cluster in a small region, so that they could deeply bias the plane determination. This is particularly true if, in virtue of item 1 , only dark bodies are involved.

The most interesting result is however that none of the planes we have found coincides with that of Sawa \& Fujimoto (2005). To better illustrate the point, using the same view angle adopted in Fig. 3 of Sawa \& Fujimoto (2005), i.e. $(l, b)=\left(296^{\circ},-11^{\circ}\right)$, in Fig. 6 we display the three planes we have derived from Eq. (7) (only one minimizes the distances of the galaxies to it) and the plane found by Sawa \& Fujimoto (2005). To add more information, in Fig. 7 we show only our best plane and that of Sawa $\&$ Fujimoto (2005). No one of our planes coincide with theirs. Why?

To strengthen our result we extend the above analysis as follows. Let us suppose that all the galaxies of the LG are located on a plane. It goes without saying that this plane contains also the vector joining MW and M 31. Now we may turn the argument around and, fixing the straight line joining MW and M 31, we may consider the manyfold of planes passing through this line. Therefore our previous analysis and result are now translated into finding the plane of the manyfold that minimizes the distances to it of all remaining galaxies. We would like to note here that the degree of freedom to our disposal (rotation of the plane around the MW-M 31 line) is not a mere mathematical problem: it is indeed due to the yet unsolved question of the tangential motion of M 31 with respect to the MW (widely discussed in Sect. 1). Similar, though not identical reasoning was

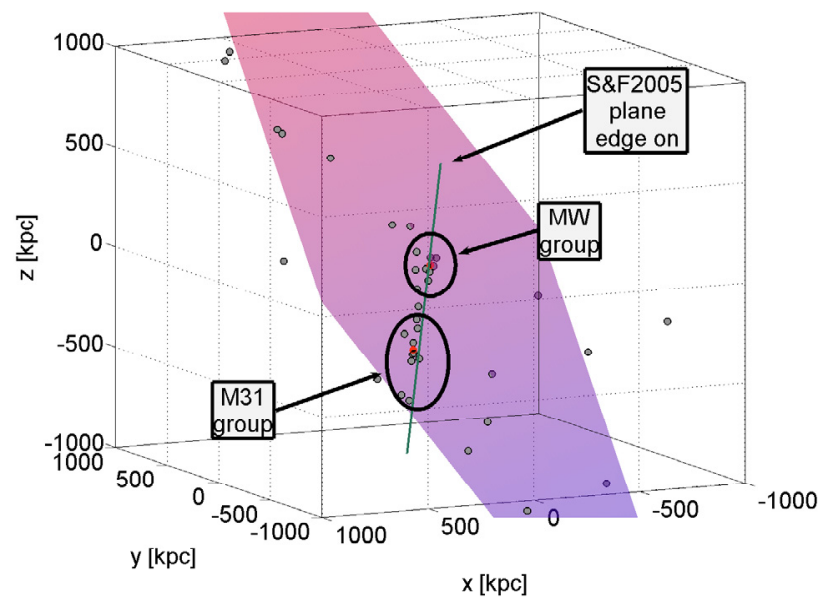

Fig. 7. 3D-view of the LG as observed from the same view angle adopted by Sawa \& Fujimoto $(2005)$, i.e. $(l, b)=\left(296^{\circ},-11^{\circ}\right)$. The hatched plane is our best plane minimizing the distances and containing also MW and M 31. The red circles are MW and M 31, the yellow circles are the dwarf galaxies above the plane, whereas the dark circles are those below it. Once again the MW and M 31 groups are indicated. The straight line is the Sawa \& Fujimoto (2005) plane seen edge-on. See the text for details.

followed by Sawa \& Fujimoto (2005) when deriving the orbits of M 31 and MW by means of the projection analysis. Fixing the plane is equivalent to reduce the degree of freedom for the motion of M 31 and to set a constraint on the dynamics of the LG as a whole (Pasetto \& Chiosi 2006, in preparation).

If the MW-M 31 vector belongs to the plane in question (reasonable assumption), the motion is mainly central and plane. Small perturbations of the central motion will be investigated in the Sect. 6.3. We are interested here to investigate the possibility that the orbital plane of the two main galaxies might be related to the plane we have just derived from mere geometrical considerations. As this result is not the rule, different determinations of the proper motion of M 31 are possible, see for instance Raychaudhury \& Lynden-Bell (1989) and Sawa \& Fujimoto (2005), depending on the kind of different physical considerations that guided the authors towards the reconstruction of the dynamical history of the LG.

The target now is to minimize the Eq. (3) imposing the constraint given by Eq. (4) and the additional one that the normal to the minimization plane has to be orthogonal to the MW-M 31 vector $\boldsymbol{a}=\left(a_{x}, a_{y}, a_{z}\right)$. The latter constraint is expressed by the relation

$\boldsymbol{n} \cdot \boldsymbol{a}=0$.

All this leads to the system of equations (the demonstration is given in Appendix A)

$$
\left\{\begin{array}{l}
2 \sum_{g=1}^{N} n_{i} x_{i}^{g} x_{j}^{g}=\lambda_{1} 2 n_{j}+\lambda_{2} a_{j} \\
n_{i} n_{i}-1=0 \\
n_{i} a_{i}=0 .
\end{array}\right.
$$

The solutions for the normals of the plane are:

$\boldsymbol{n}^{(1)}=( \pm 0.58, \pm 0.01, \pm 0.81)$

$\boldsymbol{n}^{(2)}=( \pm 0.64, \mp 0.61, \mp 0.45)$

that with the aid of the Eq. (3) give

$D^{(1)}=1.39 \times 10^{7}$

$D^{(2)}=3.11 \times 10^{6}$. 
The second solution seems to be better than the first one. This plane has the normal along the direction $(l, b)=\left(133^{\circ},-27^{\circ}\right)$.

Clearly the two planes defined by the systems of Eqs. (7) and (10) are not the same. Indeed system (10) is a simplification of system (7). However, the difference between the two planes is small as shown in Fig. 8. This amply justifies the use of the results from system (10) in the analysis below.

\section{Dynamics of the LG from the Hamilton method}

To investigate the dynamics of the LG we use the Hamilton's principle of Minimum Action. The method was developed and adapted to cosmological problems in Lagrangian description by Peebles (1989), and its applicability was tested by Peebles (1980) and Bondi (1960). The key ingredients are the boundary conditions applied to the initial and end point of each trajectory.

Each mass point (galaxy) evolves under the gravitational potential of an expanding Universe constrained by the condition

$\left\{\begin{array}{l}\delta \boldsymbol{x}_{i}(t=0) \neq 0 \wedge \lim _{a \rightarrow 0} a^{2} \dot{\boldsymbol{x}}_{i}=0 \\ \lim _{t \rightarrow t_{0}} \delta \boldsymbol{x}_{i}=0\end{array}\right.$

and the action (Peebles 1989)

$$
\begin{aligned}
S= & \int_{0}^{t_{0}}\left[\sum_{i=1}^{N_{p}} \frac{1}{2} m_{i} a^{2} \dot{\boldsymbol{x}}_{i}^{2}+\frac{G}{a} \sum_{\substack{i=1 \\
i \neq j}}^{N_{p}} \frac{m_{i} m_{j}}{\left\|\boldsymbol{x}_{i}-\boldsymbol{x}_{j}\right\|}\right. \\
& \left.+\frac{2}{3} a^{2} \pi G \rho_{b} \sum_{i=1}^{N_{p}} m_{i} \boldsymbol{x}_{i}^{2}\right] \mathrm{d} t .
\end{aligned}
$$

The original interpretation of the conditions (12) is that at the origin of the Universe the initial positions are free whereas the velocities are null. Inserting this in the hierarchical cosmological context in which the structures grow due to self-gravity from small deviations from homogeneity in an expanding Universe, the perturbations grow from different positions and null velocities. The method does not solve the equations of motion, but finds the minimum of the action (13), partially avoiding the problem of the initial conditions for the space of velocities (see Sect. 1). The only observational information that is required are the present radial velocities and positions (see Sect. 4). Owing to this, the Action Method has largely been used to study the galaxy content of the LG (Peebles 1989; Peebles et al. 1989; Peebles 1990; Dunn \& Laflamme 1993; Peebles 1995; Goldberg 2001b) or the nearby galaxies (Peebles 1994; Shaya et al. 1995; Sharpe et al. 1999; Goldberg 2001a; Peebles et al. 2001; Phelps 2002; Branchini et al. 2002; Brenier et al. 2003).

\subsection{Limitations and assumptions}

An intrinsic difficulty of the variational principle is that it does not yield the equations of motion when the action (13) is minimum, but more generally when the derivatives are null. So a path that minimizes the action (13) satisfies the equations of motion but the opposite is not true. A path that satisfies the EulerLagrange equations could not correspond to a minimum of the action (Whittaker 1989). This leads to the problem of determining the stationary points of the action integral. The issue will be addressed in the next section limited to our specific situation.

Another severe difficulty of the Action Principle concerns the mass tracers to use. The methods requires that the Baryonic Matter can be used as mass tracer and that no lumps of sole

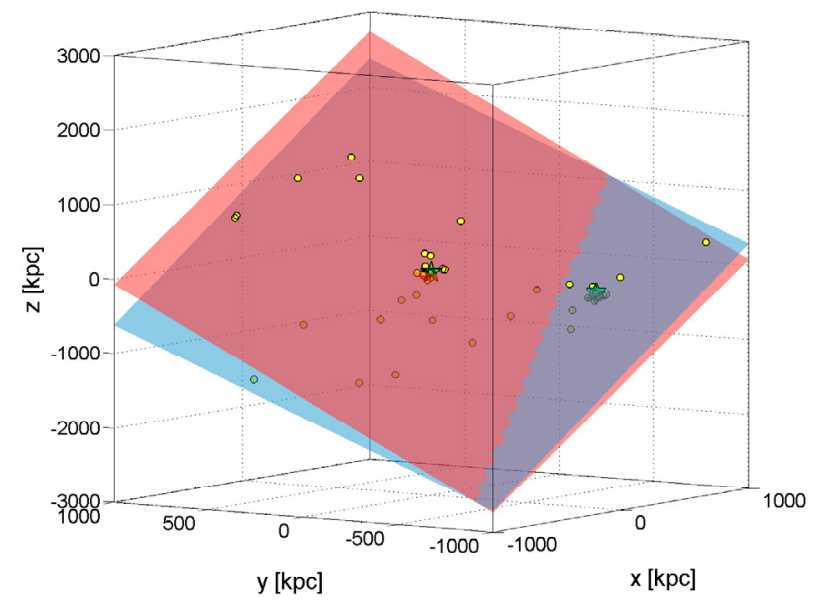

Fig. 8. The two planes minimizing the spatial distribution of galaxies in the LG. One is the best plane derived from solving the system of Eq. (5) and given by the eigen-vectors of Eq. (7) that has already been presented, the other is obtained from the system of Eqs. (9) and the eigen-vectors of Eq. (10). This second solution assumes that the vector MW-M 31 lays on the plane. Within the uncertainty the two planes coincide.

Cold Dark Matter are around altering the estimate derived from baryons. The problem has long been debated with controversial results (White et al. 1988; Davis \& Peebles 1983; Natarajan \& Springel 2004; Dunn \& Laflamme 1995).

Despite these points of uncertainty and considering that we are not interested in the direct determination of the cosmological parameters (e.g. Dunn \& Laflamme 1995), we adopt the Action method. It allows us to easily test the results for different cosmological models.

In the general case of a cosmologically flat Universe, the curvature of the Universe is given by

$\frac{\dot{a}}{a}=H_{0}\left[\Omega_{0}(1+z)^{3}+\Omega_{\Lambda}\right]^{1 / 2}$

where $H_{0}$ is the Hubble constant, and $\Omega_{0}$ and $\Omega_{\Lambda}$ the density parameters. The corresponding orbital equations can be written as

$$
\begin{array}{r}
a^{1 / 2} \frac{\mathrm{d}}{\mathrm{d} a}\left(a^{3 / 2} \frac{\mathrm{d} \boldsymbol{x}_{i}}{\mathrm{~d} a}\right)+\frac{3 a^{4}\left(1-\Omega_{0}\right)}{2 F_{3}} \frac{\mathrm{d} \boldsymbol{x}_{i}}{\mathrm{~d} a}= \\
\frac{\Omega_{0}}{2 F_{3}}\left[\frac{R_{0}^{3}}{M_{T}} \sum_{\substack{j=1 \\
j \neq i}}^{N_{p}} \frac{m_{j}\left(\boldsymbol{x}_{i}-\boldsymbol{x}_{j}\right)}{\left\|\boldsymbol{x}_{i}-\boldsymbol{x}_{j}\right\|^{3}}+\boldsymbol{x}_{i}\right]
\end{array}
$$

where $R_{0}$ is the radius at $t=t_{0}$ of a sphere containing the total mass $M_{T}$ of the galaxies in the solution assuming an homogeneous mass density. The quantity $F_{3}$ is derived from the relation

$F_{p} \equiv \Omega_{0}+a^{p}\left(1-\Omega_{0}\right)$

with $p=1$ in the case of $\Lambda=1$ and $p=3$ in the case of $\Lambda \neq 0$. They will be used here only to check the compatibility with the results derived from the Minimum Action (13).

\subsection{Analytical representation of the orbits}

A suitable representation of the orbits to be inserted in the system (15) has been proposed by Peebles (1989). They are 
expressed as a linear combination of suitable universal functions of time with unknown coefficients $\boldsymbol{C}_{i, n}$

$\boldsymbol{x}_{i}(a)=\boldsymbol{x}_{i}\left(t_{0}\right)+\sum_{n=0}^{N-1} \boldsymbol{C}_{i, n} f_{n}(a)$

for all $i=1 \ldots N_{p}$, where $\boldsymbol{x}_{i}\left(t_{0}\right)$ are the present positions of the galaxies, $N_{p}$ the number of galaxies or clusters, and $f_{n}(a)$ the basis functions able to satisfy the boundary conditions (12). Classical expressions for the $f_{n}(a)$ are either

$f_{n}=a^{n}(1-a)$

that is suggested by Peebles (1989) or

$f_{n}(1-a)^{s-n} a^{n}\left(\frac{s !}{n !(s-n) !}\right) \wedge n \in[0, n[$

that is proposed by Shaya et al. (1995), where $s$ is a parameter and $n<s$.

Another useful expression is the one proposed by Giavalisco et al. (1993) who assume that the series representing the quasilinear solution has to be as close as possible to the linear solution and to converge to it in the limit of small perturbations. Therefore the leading term of Eq. (17) is the linear approximation of Zel'dovich (e.g. Coles \& Lucchin 2002). This approximation holds good only in the earliest stages of the Universe due to its linear character. However it has widely been used to describe density perturbations over large scales, treating galaxy clusters as particles. This is possible because the strongly non linear motions inside a cluster (LG as well as other nearby groups) somewhat balance each other, so that the motion of the barycentre is influenced only by its closest neighbourhood. Thanks to it we may adopt the equations

$\boldsymbol{x}_{i}(t)=\boldsymbol{x}_{i, 0}+\sum_{n=1}^{N}\left(D(t)-D_{0}\right)^{n} \boldsymbol{C}_{n, i}$,

where the linear-growth function $D$ is taken from Heath (1977) and $D_{0}$ is

$D_{0}=D\left(t_{0}\right)=\left[\frac{\dot{a}}{a} \int_{0}^{a} \frac{\mathrm{d} a}{\dot{a}^{3}}\right]_{a_{0}}$.

Finally, another approach has been developed by Nusser \& Branchini (2000) using the so-called Fast Action Minimization Method. In brief, a Tree-Code algorithm quickly computes the gravitational field contained in the action (13) and the solution (17) is expanded as a function of the growth factor $D$ (Heath 1977) and the derivative of the solution (17). This procedure is not used here due to the small number of galaxies we are dealing with (see Sect. 4).

As there are many different minima of the action (13) able to reproduce a given set of initial conditions, another version of the least action method has recently been proposed by Goldberg \& Spergel (2000) and applied to reconstruct the haloes of MW and M 31 (Goldberg 2001b) considered as isolated systems.

As explained in Sect. 5 we have tried different algorithms to check the results originating from different minima of the action.

\section{Neighbourhood of the LG: their dynamical effects}

To consider the LG as an isolated system, the forces exerted by the external galaxies or groups must be lower than those developed by the internal galaxies. Typically an external mass $M$ at a distance $\delta$ will produce a force for unitary mass $\sim G M / \delta^{2}$ if it is sufficiently spherical so that multi-pole contribution can be neglected. The mutual forces that MW and M 31 exert each other have two main contributions: a mean force produced by distant bodies (see Table 2) that is is the target of this study) and a fluctuating force due to the dwarf galaxies inside the LG (Pasetto \& Chiosi 2006, in preparation). To give a rough idea of the problem, let us consider the LG as made by a number $N$ of galaxies of mass $M_{\mathrm{g}}$, assumed to be uniformly and spherically distributed. At any distance $r$ from the centre of the LG the inner total mass $M_{\mathrm{LG}}(r)$ is given by

$M_{\mathrm{LG}}(r)=N M_{\mathrm{g}}\left(\frac{r}{R_{\mathrm{LG}}}\right)^{3}$,

where $R_{\mathrm{LG}}$ is the total radius of the LG. This produces a mean force field

$\left\langle\frac{G M_{\mathrm{LG}}(r)}{r^{2}}\right\rangle \approx \frac{G N M_{\mathrm{g}}}{R_{\mathrm{LG}}^{3}} r$.

Therefore, the mean internal force will be greater than the external one if the following condition is satisfied

$\frac{M_{\mathrm{LG}}}{M}>\frac{R_{\mathrm{LG}}^{3}}{\delta^{2} r}$

Substituting $r$ with $R_{\mathrm{LG}}$ we get $R_{\mathrm{LG}}^{2} M / \delta^{2} M_{\mathrm{LG}}<1$ which, using the entries of Table 2 and the standard values of $R_{\mathrm{LG}} \approx 1.2 \mathrm{Mpc}$ and $M_{\mathrm{LG}} \approx 2.5 M_{\odot}$ (see e.g. van den Bergh 1999; Courteau \& van den Bergh 1999) falls in the range 0.2 to 0.5. This confirms that the external galaxies do not dominate the internal dynamics of the LG. However they could have played an important role either in the past as shown by Dunn \& Laflamme (1993) or even at the present time if one pushes the analysis to a deeper level trying to establish the correlation between torsion and quadrupole moments of the gravitational field when the assumption of spherical symmetry is relaxed ${ }^{2}$

$\frac{\mathrm{d} \boldsymbol{I}_{\mathrm{im}} \omega_{\mathrm{m}}}{\mathrm{d} t}=-\frac{1}{3} \sum_{j, k} \varepsilon_{i j k} q_{j l} Q_{l k}$,

where, $\boldsymbol{I}_{\mathrm{im}}$ is the inertia momentum tensor of the LG's galaxy, $\omega_{\mathrm{m}}$ its frequency of rotation in approximation of rigid body, $q_{\mathrm{jl}}$ the quadrupole moment of the selected galaxy, and $Q_{\mathrm{lk}}$ the quadrupole components of the external gravitational field. This is suggested by the planar distribution we have found and the results obtained by Raychaudhury \& Lynden-Bell (1989) and Peebles et al. (2001), who followed the dynamical evolution of the LG galaxies (see Table 1) considering the influence of external galaxies and groups. It is worth commenting here that the concept of LG itself becomes meaningful only when the two major galaxies are so deeply influencing one another that both the quadrupole terms and the torsion are of internal origin (Dunn \& Laflamme 1993). Therefore, the evaluation of the dynamical effects exerted by the dominant galaxies in the LG itself and external galaxies and groups is a mandatory, preliminary step to be undertaken.

The major source of data is the NBG together with many subsequent implementations. To mention a few, we recall Peebles (1989), Raychaudhury \& Lynden-Bell (1989), Peebles (1990),

2 The contribution to the force field due to the LG dwarf galaxies however cannot be estimated in the same way because the fluctuations are comparable to the instantaneous value. 
Dunn \& Laflamme (1993), Peebles (1994, 1995), Sharpe et al. (1999), Peebles et al. (2001), and references.

The key data to use here are the distances and the radial velocities. The uncertainty affecting the distances is particularly relevant here because it influences very much the derivation and prediction of a galaxy orbit and its evolution in turn. Thoughtful analyses of the importance of good distances in tracking-back the orbits of the LG galaxies are by Valtonen et al. (1993) and Byrd et al. (1994) to whom the reader should refer for details. Their reconstruction of the orbits of MW and M 31 is based on the distances of Maffei 1 (Buta \& McCall 1983) and IC 342 (McCall 1989) (based in turn on recent determinations of the Galaxy extinction). In these studies, the highest uncertainty cames from the distance of Maffei 1 . The authors assumed $1.8 \mathrm{Mpc}$, much shorter than $3.9 \mathrm{Mpc}$ given by Tully \& Fisher (1988) and the recent determination of $3.5 \mathrm{Mpc}$ reported by Fingerhut et al. (2003).

Over the years many efforts have been made to cope with the uncertainty due to distances. Recently Phelps (2002) showed that a canonical transformation of the action (13) can be used to work directly with the natural boundary conditions at redshift $z=0$ and the angular position $\left(l^{\circ}, b^{\circ}\right)$ of a moving observer. Similar attempts have been made either by changing the orbit functions (Giavalisco et al. 1993) or the action (Schmoldt \& Saha 1998), or using the method of variable end-points and the "transversality" condition (Whiting 2000).

In the following we will select a sample of external galaxy groups for which we will evaluate the dynamical effects on the LG. Fortunately the uncertainty on the distances for this sample are found not to significantly affect our results. In any case we will always keep in mind that distances are the crucial parameter of our analysis and observational astronomy in general, see e.g. Carrasco et al. (2004) and Walker (2003).

Given these premises, we briefly review the main properties of the most massive galaxies or galaxy groups in both the LG itself and the surroundings that could dynamically affect the LG. These are:

1. IC 342 group: this is the major spiral galaxy of a small group containing also NGC 1560 and UGCA 105. Recently Saha et al. (2002) improved upon the distance using 20 Cepheid stars and derived the distance modulus $27.58 \pm 0.18$, i.e. a distance of $3.3 \mathrm{Mpc}$. For this group of galaxies we adopt the total mass of $1.26 \times 10^{13} M_{\odot}$ and the radial velocity of $171 \mathrm{~km} \mathrm{~s}^{-1}$ quoted by Peebles (1994).

2. Maffei group: two galaxies are known under this name, Maffei 1 and Maffei 2. Recent determinations of the distance go from $3 \mathrm{Mpc}$ of Fingerhut et al. (2003) to $4 \mathrm{Mpc}$ of Peebles (1989). We adopt here the mean value of 3.5 Mpc as found in Dunn \& Laflamme (1993). The total mass of the pair is $6.32 \times 10^{12} M_{\odot}$. The radial velocity from the $\mathrm{MW}$ is $152 \mathrm{~km} \mathrm{~s}^{-1}$ (Peebles 1989).

3. M 31 group: Under this name we consider the group containing M 31, M 33, IC 10 and LGS3. The radial velocities are taken from Peebles (1989). The adopted total mass mass is $3.16 \times 10^{12} M_{\odot}$. A smaller mass for the halo of M 31 has been suggested by Evans et al. (2000). It will be taken into consideration when testing the model results in Sect. 6 .

4. M 81 group: this contains NGC 2366, NGC 2976, NGC 4236, IC 2574, DDO 53, DDO 82, DDO 165, Holmberg I, Holmberg II, Holmberg IX, K52, K73, BK3N, Garland, and A0952+69. Recent determinations of the kinematics, distances and structures are by Karachentsev et al. (2002). The total mass is $1.6 \times 10^{12} M_{\odot}$,
Table 2. External galaxy groups gravitationally influencing the LG dynamics.

\begin{tabular}{cccccc}
\hline \hline $\begin{array}{c}\text { Group } \\
\text { name }\end{array}$ & $\begin{array}{c}l \\
\circ\end{array}$ & $\begin{array}{c}b \\
\circ\end{array}$ & $\begin{array}{c}d \\
\mathrm{Mpc}\end{array}$ & $\begin{array}{c}M \\
10^{12} M_{\odot}\end{array}$ & $\begin{array}{c}V_{r} \\
\mathrm{~km} \mathrm{~s}^{-1}\end{array}$ \\
\hline IC 342 & 138.2 & 10.6 & 3.30 & 12.6 & 171.0 \\
Maffei & 136.4 & -0.4 & 3.50 & 6.3 & 152.0 \\
Andromeda & 121.2 & -21.6 & 0.76 & 3.16 & -123.0 \\
Milky Way & 0 & 0 & 0.0 & 2.2 & 0.0 \\
M 81 & 142.1 & 40.9 & 3.7 & 1.6 & 130.0 \\
Cen A & 309.5 & 19.4 & 2.7 & 4.7 & 371.0 \\
Sculptor & 105.8 & 85.8 & 3.2 & 6.3 & 229.0 \\
\hline
\end{tabular}

The radial velocity is referred to the centre of the MW. No correction for the motion of the Sun toward the Local Standard of Rest is applied because it falls below the accuracy adopted in this study. The uncertainties on the distances and radial velocities are omitted.

the mean distance is $3.7 \mathrm{Mpc}$ and the radial velocity is $130 \mathrm{~km} \mathrm{~s}^{-1}$.

5. Cantaurus A (NGC 5128) group: for the group made of NGC 5128 and NGC 4945 we adopt the results of Mould et al. (2005). The distance is $2.7 \mathrm{Mpc}$, the total mass is $4.47 \times$ $10^{12} M_{\odot}$ and the radial velocity is $371 \mathrm{~km} \mathrm{~s}^{-1}$.

6. Sculptor (NGC 253) group: this contains NGC 253, NGC 247, NGC 55 and NGC 7793 (Dunn \& Laflamme 1993). The total mass is $6.32 \times 10^{12} M_{\odot}$, the radial velocity is $152 \mathrm{~km} \mathrm{~s}^{-1}$. The distance we have adopted is $3.2 \mathrm{Mpc}$ (Dunn \& Laflamme 1993).

7. M 83 (NGC 5236) group: the recent study of Thim et al. (2003) with the ESO VLT yields the distance of $4.5 \mathrm{Mpc}$ and the radial velocity of $249 \mathrm{~km} \mathrm{~s}^{-1}$. We adopt the total mass of $7.99 \times 10^{11} M_{\odot}$.

To these groups of galaxies we have to add of course the MW. The basic data for this sample of galaxy groups are given in Table 2, which lists the galacto-centric coordinates $l$ and $b$, the distance, the mass and the radial velocity. The radial velocities adopted here have been cross-checked with the data in the catalog by Karachentsev (2005). The differences are taken into account in the statistical analysis of the results to be presented in Sect. 6 below. We may anticipate that at the accuracy level of the present study no significant differences are found.

These are the eight dominant galaxy groups. They contains about 90 per cent of all the light and so, if we assume that the light is a tracer of the mass, as argued in Sect. 3, these are the key objects whose motion has to be investigated in order to derive the force field acting on the LG. The analysis will be made in two steps: (i) first we will consider only the eight objects listed in Table 2; (ii) second, following Peebles et al. (2001), we will increase the sample adding a number of galaxies of smaller mass. The new list is shown in Table 3. This would allow us to estimate the dependence of the results on the number of objects under consideration or in other words to evaluate how small mass objects "perturb" the orbits of the large mass ones.

\section{Minimizing the action}

The main goal of this section is to provide a physical explanation for the planar distribution of the galaxies in the LG found in Sect. 2. With the least action principle (Sect. 3) we want to estimate the influence of the external galaxy groups (see Table 2) on the LG (see Table 1) and look for a dynamical justification of the planar distribution we have found from the mere geometrical analysis. Knowing the external force field acting on the LG could 
Table 3. Complementary sample of low mass galaxies.

\begin{tabular}{cccccc}
\hline \hline Galaxy & $l$ & $b$ & $d$ & $V_{r}$ & $M$ \\
\hline N2403 & 149.6 & 29.3 & 3.3 & 227 & 11.8 \\
N4236 & 12.3 & 47.3 & 3.9 & 118 & 11.5 \\
Circinus & 311.3 & 3.7 & 3.3 & 268 & 11.5 \\
N55 & 332.6 & -75.6 & 1.6 & 96 & 11.5 \\
N7793 & 4.4 & -77.1 & 4.1 & 230 & 11.5 \\
N300 & 299.2 & -79.4 & 2.1 & 101 & 11.3 \\
N1569 & 143.6 & 11.2 & 2.4 & 47 & 11 \\
N404 & 126.9 & -27.1 & 3.6 & 115 & 10.8 \\
N3109 & 262.1 & 23.0 & 1.3 & 194 & 10.5 \\
IC5152 & 343.8 & -50.1 & 1.7 & 85 & 10 \\
SexA,B & 240.3 & 42.0 & 1.4 & 165 & 10 \\
N6822 & 25.3 & -18.3 & 0.5 & 45 & 10 \\
N1311 & 264.2 & -52.6 & 3.1 & 425 & 9.8 \\
IC1613 & 129.7 & -60.5 & 0.7 & -155 & 9.8 \\
WLM & 75.8 & -73.6 & 0.9 & -65 & 9.7 \\
VIIZw403 & 127.8 & 37.3 & 4.5 & 50 & 9.6 \\
SagDIG & 21.0 & -16.5 & 1.1 & 7 & 9.1 \\
PegDIG & 94.7 & -43.5 & 0.8 & -22 & 8.9 \\
LeoA & 196.8 & 52.4 & 0.7 & -18 & 8.8 \\
DDO210 & 34.0 & -31.3 & 0.9 & -24 & 8.7 \\
DDO155 & 310.8 & 76.9 & 1.5 & 183 & 8.6 \\
Phoenix & 272.0 & -68.9 & 0.4 & -34 & 7.7 \\
\hline
\end{tabular}

This sample is added to the sample of Table 2 . The effect of these galaxies can be considered as a perturbation. The coordinates $l$ and $b$ are in ${ }^{\circ}$, the distances $d$ are in Mpc (as in the previous table we omit the uncertainties on the distances and velocities), the radial velocity $V_{r}$ is in $\mathrm{km} \mathrm{s}^{-1}$, and the galaxy masses are in solar units. They are expressed as $\log M$.

indeed cast light on whether the planar distribution is a temporary situation holding only at the present time $t=t_{0}$, whereas the galaxy phase-space could tell a different story for all ages different from $t_{0}$.

To this aim, using the galaxies of Table 2, we minimize the action for an unconstrained Lagrangian given by Eq. $(13)^{3}$.

We adopt the standard cosmological scenario with cosmological constant and the condition $\Omega_{0}+\Omega_{\Lambda}=1$ we have already shown in Eq. (14). The standard cosmological model is for $H_{0}=72 \mathrm{~km} \mathrm{~s}^{-1} \mathrm{Mpc}^{-1}, \Omega_{0}=0.3$ and $\Omega_{\Lambda}=0.7$. The orbits of the eight dominant groups together with the MW and M 31 are followed from redshift $z=60$ till now. The orbits resulting from the minimization procedure are presented in Fig. 9.

To avoid solutions with overlapping orbits that cannot be treated with the technique of polynomial expansion of the orbits as a function of the Universe expansion parameter, we have introduced a smoothing length $\epsilon$ in the gravitational potential. The acceleration acting on the $i$ th particle and measured by a comoving observer at rest respect to distant matter is given by

$\boldsymbol{g}_{i}=\frac{G}{a^{2}} \sum_{\substack{j=1 \\ j \neq i}}^{N_{p}} \frac{m_{j}\left(\boldsymbol{x}_{i}-\boldsymbol{x}_{j}\right)}{\left\|\left(\boldsymbol{x}_{i}-\boldsymbol{x}_{j}\right)^{2}+\varepsilon^{2}\right\|^{3 / 2}}+\frac{4}{3} a \pi G \rho_{b} \boldsymbol{x}_{i}$

where the smoothing length we have adopted is from Peebles (1990), i.e. $\epsilon \cong 150 \mathrm{kpc}$.

The smoothing length is the lower limit below which the analysis cannot be pushed, and hence the maximum resolution of the plane thickness. This is dynamically extremely relevant because it allows us to avoid un-physical solutions in the minimization of the action. Indeed the case of two orbits crossing

3 A different analysis based on the orbit integrations and proper motions of LG galaxies is in preparation (Pasetto \& Chiosi 2006).

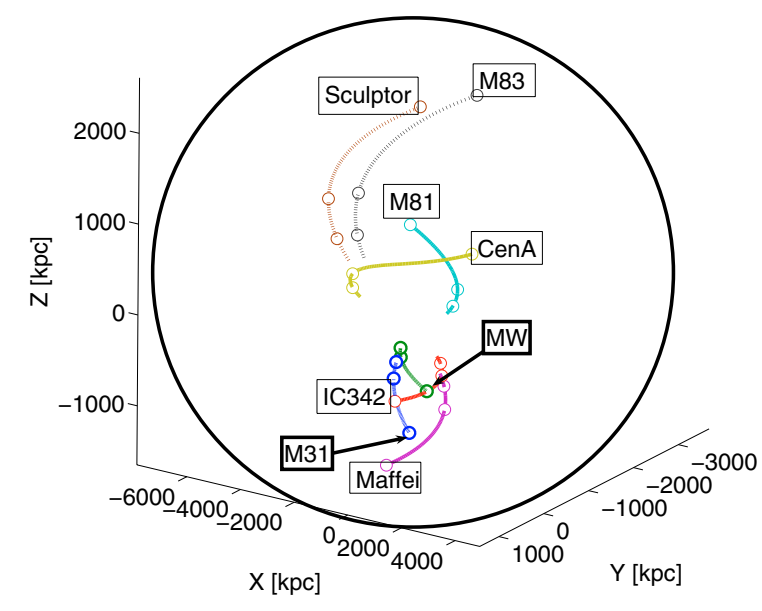

Fig. 9. Orbits of the eight dominant galaxies or groups of galaxies derived from the minimization of the unconstrained action of Eq. (13). The orbits are shown as in Peebles (1989) for the sake of comparison. The orbits of MW and M 31 are highlighted. The circle has a radius $R_{0}=3.5 \mathrm{Mpc}$, see Eq. (15) and the text for more details. We also tested the approximation adopted for the analytical representation of the orbits given by Eq. (17) with the equation of motion of Eq. (15). The corresponding orbits are nor shown as they in practice overlap with the present ones. Along each orbit a few selected stages are displayed: the initial stage at $z=60$, the stage at $z=2$ (the first circle), the stage at $z=1$ (the second circle) and the stage at the present time $z=0$ (the third circle).

each other would inevitably lead to a merger, which cannot be properly treated by the technique in use here. Fortunately, it has recently been proved that orbit crossing events are less of a problem as far as the number of solutions able to minimize the action (13) is concerned (Whiting 2000). In other words, this means that the dynamics we are going to analyse is collisionless. It is worth noting that this description may not hold at the earliest stages of the Universe (redshift $z \cong 60$ ) when the galaxy sizes were likely comparable to their mean separation (Dunn \& Laflamme 1995). However, the approach we have adopted can be justified in statistical sense: if we consider our galaxy groups (and dominant galaxies) as mass point, each with dimension $d \approx \varepsilon$, the smoothing length, the system of eight point-like galaxy groups can be considered isolated. This is because the volume occupied by the their orbits, $\sim 2000^{3} \mathrm{kpc}^{3}$ (see Fig. 9), is greater than the total volume occupied by the objects, $\sim \varepsilon^{3}=150^{3} \mathrm{kpc}^{3}$. Two objects of radius $\varepsilon$ have an effective radius of $2 \varepsilon$ and a cross section $\sigma=4 \pi \varepsilon^{2}$. In the very early stages of the Universe, assuming uniform distribution and random motion for the $N$ objects of Table 2, we can define the mean free path as $\lambda=\bar{v} / \bar{V} n \sigma$, where the relative mean velocity of the LG members is nearly equal to the mean velocity of the group itself, $\bar{V} \geq \bar{v}$. Therefore, the mean free path of collisions is $\lambda \approx 1 / n \sigma=R^{3} / 3 N \varepsilon^{2}$, about $15 \mathrm{Mpc}$ for the eight massive objects of Table 2, i.e. larger than the dimensions we are interested in. This means that we expect collisions to rarely affect the motion of the objects in the sample (but see e.g. Diemand et al. 2005; Diemand 2005). The same considerations cannot be extended to the total sample given by Tables $1-3$ because of the very large range of masses spanned by the objects. It is worth recalling here that the constraints given by Eq. (12) refer to the present time, whereas back in the past $(z=60)$ the positions were random and the velocities null. In other words, but for the very initial stages, as soon as the collisionless description can be 
Table 4. Velocities and positions of the galaxies used in the minimization procedure.

\begin{tabular}{ccccccc}
\hline \hline $\begin{array}{c}\text { Galaxy } \\
\text { name }\end{array}$ & $\begin{array}{c}v_{x}^{\mathrm{G}} \\
\mathrm{km} \mathrm{s}^{-1}\end{array}$ & $\begin{array}{c}v_{y}^{\mathrm{G}} \\
\mathrm{km} \mathrm{s}^{-1}\end{array}$ & $\begin{array}{c}v_{x}^{\mathrm{G}} \\
\mathrm{km} \mathrm{s}^{-1}\end{array}$ & $\begin{array}{c}x^{\mathrm{G}} \\
\mathrm{kpc}\end{array}$ & $\begin{array}{c}y^{\mathrm{G}} \\
\mathrm{kpc}\end{array}$ & $\begin{array}{c}z^{\mathrm{G}} \\
\mathrm{kpc}\end{array}$ \\
\hline IC 342 & -294.7 & 80.0 & -21.1 & 1309.6 & 1176.0 & -368.2 \\
Maffei & -359.1 & 97.1 & -66.1 & 1426.0 & 1427.6 & -999.7 \\
Andromeda & 125.5 & -25.9 & -85.1 & -742.5 & -381.6 & -1255.0 \\
Milky Way & 117.3 & -67.0 & -65.7 & -1108.5 & -986.0 & -975.3 \\
M 81 & -391.3 & 49.8 & 97.4 & 1098.3 & 731.9 & 1447.3 \\
Cen A & 725.4 & -200.7 & 83.4 & -2728.4 & -2951.1 & -78.4 \\
Sculptor & 822.1 & -51.7 & 149.5 & -1044.7 & -760.5 & 2216.2 \\
M 83 & 618.0 & -250.6 & 89.1 & -3807.0 & -3684.5 & 1409.4 \\
\hline
\end{tabular}

The masses have been taken from Table 2 . The positions are referred to the barycentre of the sample located at $\boldsymbol{x}_{\mathrm{G}}=\{1117,986,975\} \mathrm{kpc}$.

applied, the results we have presented are a good description of the real galaxy motions.

Finally, the motions can be referred to the barycentre of the system to simply and correctly follow the Universe expansion. The new positions and velocities are given in Table 4 (and in Fig. 9). For instance, referred to the barycentre of the group $\boldsymbol{x}_{\mathrm{G}}=[1117,986,975] \mathrm{kpc}$, the velocity vector of M 31 at the present time is $\boldsymbol{v}_{\mathrm{M} 31}\left(t_{0}\right)=[125.5,-25.9,-85.0] \mathrm{km} \mathrm{s}^{-1}$ and the projection of the velocity vector onto the line joining the barycentre to the M 31 position is $\boldsymbol{v}_{\text {rad,M } 31}^{(\mathrm{G})}\left(t_{0}\right)=-15.4 \mathrm{~km} \mathrm{~s}^{-1}$. This is one of the many possible velocity vectors matching this radial projection, however, it is the only one minimizing the action of Eq. (13).

This kind of analysis based on the same sample of Table 2 has already been made by Dunn \& Laflamme (1993) to derive the proper motion of M 31 .

At this stage, an interesting question can be posed. Is there any correlation between the plane found in Sect. 2 and the action minimization? In other words how would the action minimum change if the plane is added as constraint to the whole procedure?

Using the representation of the plane given by Eq. (10), we impose that the positions of M 31 and MW are those given by the observations, the same for the radial velocity of $\mathbf{M} 31$, and that the present-day velocity vectors of the two galaxies belong to the plane. From a dynamical point of view, the latter hypothesis is not equivalent to that adopted by Sawa \& Fujimoto (2005). They assumed a central \& plane motion for the LG galaxies and derived the dynamical history of the LG. Here no assumption is made neither on the angular momentum or torsion of the LG, nor the quadrupole-quadrupole interaction, nor the inertia tensor of our main galaxies (see Eq. (25) and Dunn \& Laflamme (1993) for similar considerations). Finally, owing to the expected minimum thickness of the theoretical plane caused by the smoothing length, we allow for the possibility that the velocity vector cannot to exactly belong to the plane, although very close to it. From a mathematical point of view, this means that a anholonomic constraint has to be chosen instead of the pure holonomic one used in Sect. 2. Therefore the holonomic constraint evaluated only at the time $t=t_{0}$ and expressed by

$v_{x} n_{x}+v_{y} n_{y}+v_{z} n_{z} \cong 0$

is replaced by the more suitable condition

$\left\|a\left(\frac{\mathrm{d} x}{\mathrm{~d} a} n_{x}+\frac{\mathrm{d} y}{\mathrm{~d} a} n_{y}+\frac{\mathrm{d} z}{\mathrm{~d} a} n_{z}\right)+n_{x} x+n_{y} y+n_{z} z\right\| \leq \varepsilon$,
Table 5. Velocities derived from the Constrained Minimum Action.

\begin{tabular}{cccc}
\hline \hline $\begin{array}{c}\text { Galaxy } \\
\text { name }\end{array}$ & $\begin{array}{c}v_{x}^{\mathrm{G}} \\
\mathrm{km} \mathrm{s}^{-1}\end{array}$ & $\begin{array}{c}v_{y}^{\mathrm{G}} \\
\mathrm{km} \mathrm{s}^{-1}\end{array}$ & $\begin{array}{c}v_{z}^{\mathrm{G}} \\
\mathrm{km} \mathrm{s}^{-1}\end{array}$ \\
\hline IC 342 & -297.6 & 83.2 & -24.5 \\
Maffei & -352.2 & 94.7 & -61.8 \\
Andromeda & -22.8 & -26.0 & 2.7 \\
MW & 7.1 & 55.7 & -64.5 \\
M 81 & -387.1 & 48.8 & 97.0 \\
Cen A & 722.4 & -215.1 & 79.2 \\
Sculptor & 827.1 & -50.1 & 142.9 \\
M 83 & 622.1 & -245.4 & 88.7 \\
\hline
\end{tabular}

The positions are taken from literature and are left unchanged. See the text for more details.

where $\varepsilon \rightarrow 0$. This is not a genuine anholonomic constraint because it has to be satisfied only at the extremal points of all possible trajectories and not along the whole path, i.e.

$\left\{\begin{array}{l}\delta \boldsymbol{x}_{i}(t=0) \neq 0 \wedge \lim _{a \rightarrow 0} a^{2} \dot{\boldsymbol{x}}_{i}=0 \\ \lim _{t \rightarrow t_{0}} \delta \boldsymbol{x}_{i}=0 \wedge \hat{\boldsymbol{n}} \cdot \boldsymbol{x}_{i}\left(t=t_{0}\right)=0 .\end{array}\right.$

This assumption allows us to avoid more complicated minimization procedures, e.g. Saletan \& Cromer (1970) for the Constrained Minimum Action Principle.

The solution for the minimization is shown in Fig. 10 and the companion Table 5. In general the velocities remain the same as before but for M 31 and MW for which they significantly change. This simply reflects the constraint that at $t=t_{0}$ the two galaxies in the phase space belong to the manifold expressed by the conditions (12) and (27). The most interesting and important result is that now the action is significantly lower than in the previous case. We suspect that profound implications are hidden in this result. We may provisionally conclude that the orbits of $M W$ and $M 31$ lie on a plane and that this likely coincides with that derived from the geometrical analysis.

Before proceeding further, there are a number of considerations to be made here:

- As both the Lagrangian of the action integral (13) and the set of galaxies in use (Table 2) are let unchanged, the space of possible constraints of the first minimization must contain also the one of the second minimization. Therefore, in the first case the procedure has not been able to locate the absolute minimum because of the large dimensions of the minimization space (roughly 120 dimensions). The minimum found in the first case can be considered either as a local minimum or a saddle. The saddle situation is a classical problem with this kind of minimization and often masks the true solution (see e.g. Brenier et al. 2003; Peebles 1994, and the case of NGC 6822 orbit). This somewhat proves that the second minimization is more correct than the first one.

- The attempts to get the motion of M 31 based on this methods cannot give physically sounded results without an adequate estimate of the errors. Therefore, the proper motion derived by Dunn \& Laflamme (1993) and Sawa \& Fujimoto (2005) has to be considered as one of the possible values. We will touch upon this point in more detail testing the stability of our results (see below).

- Velocity vectors belonging to the plane of MW, M 31 and all other dwarf galaxies of the LG correspond to a solution more realistic than the one given by the un-constrained minimization. This does not, however, ensure that the solution is stable. Even assuming that in the LG the force field is 


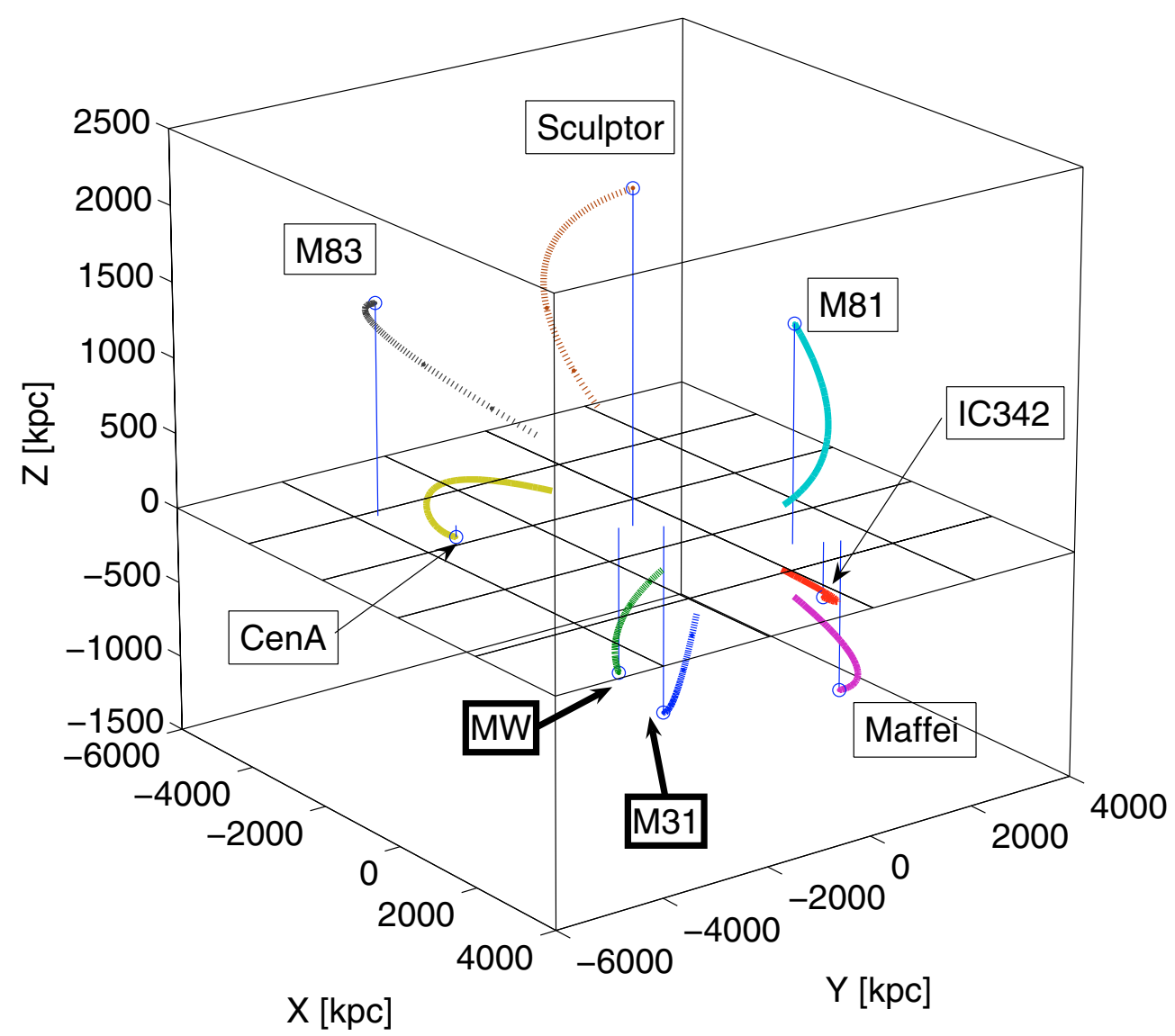

Fig. 10. Orbits of the galaxy groups and the two dominant galaxies derived from the constrained minimization of the action. The constraints are given by relations (27) and (28). The dots and circles along each orbit have the same meaning as in Fig. 9. The circles correspond to the present age.

dominated by the binary interaction between MW and M 31, this does not automatically exclude that the two galaxies are simply undergoing a fly-by encounter and that they will not depart from each other in the future. The existence of a $d y$ namically justified plane must be proved by the analysis of the external forces acting on the LG at all ages.

In the section below we will examine in detail the robustness of the result just obtained and the implications for the force field acting on the galaxies of the LG.

\section{Robustness of the solution}

We proceed now to test the solution we have obtained as far as three points of weakness are concerned.

1. The minimization method. How much the result depends on the minimization method in use? To answer this question first we adopt three different minimization algorithms, second we change the random number generator and repeat the analysis several times because in the so-called optimization problems in multidimensional spaces one deals with statistical results that have to be tested against the adopted statistical method (see below).

2. Orbit representation. The analytical representation of the orbits used in Eqs. (13) and (15) to obtain the final velocities is a critical aspect of the whole problem. Like in other studies, (see for instance Peebles 1989; Dunn \& Laflamme 1993), the orbit traced-back by the action (13) agree with those derived with the equation of motion (15). However, the tangent to the orbits at $a=1$, i.e. $t=t_{0}$, strongly depends on the number of terms considered in the series expansion of Eq. (18). To be physical meaningful, the result must be independent of analytical expressions in use. Therefore, we will check whether the results remain the same using Eq. (20).

3. Number of galaxies in the sample. Finally, it is worth testing whether the results vary with the number of galaxies that are taken into account. To this aim we re-determine the velocities for the extended set of galaxies which includes also all the objects listed in Table 3.

\subsection{Minimization methods}

In this subsection we test the possible dependence of the results from the minimization method. In literature there are several algorithms of optimization in multidimensional spaces. In general, a great advantage is possible if the gradient in the action (13) can be calculated

$\nabla_{\boldsymbol{C}_{i, n}} S=m_{i} \int_{0}^{t_{0}}\left[-\frac{\mathrm{d}}{\mathrm{d} t}\left(a^{2} \dot{\boldsymbol{x}}_{i}\right)+a \boldsymbol{g}_{i}\right] f_{n} \mathrm{~d} t$,

where the gravitational acceleration is given by Eq. (26). This possibility makes the methods based on the nonlinear conjugate gradient (Press et al. 1986) very efficient to derive an unconstrained optimization. Alternatively, for a small sample of 
galaxies, one can invert the matrix of the second derivatives as described in Peebles (1995). This was indeed the first method to be adopted. However, there are other methods that are suited to the aims and are also widely used in literature. In the following we apply some of them to our problem.

- Differential evolution. This is a widely used genetic algorithm (Storn \& Price 1995; Fleiner 1997). It stands on a series of guess vectors, $\boldsymbol{x}_{1}, \ldots, \boldsymbol{x}_{n}$, of real numbers (genes). At each iteration, each $\boldsymbol{x}_{i}$ randomly selects the integers $\{a, b, c\}$ to build up the mate $\boldsymbol{y}_{i}=\boldsymbol{x}_{a}+\gamma\left(\boldsymbol{x}_{b}-\boldsymbol{x}_{c}\right)$ where $\gamma=1 / 2$ is a scaling factor. Then $\boldsymbol{x}_{i}$ is linked to $\boldsymbol{y}_{c}$ according to the value of the crossing probability (constantly equal to $1 / 2$ ) producing the $z_{c}$. Finally $\boldsymbol{x}_{i}$ competes against $z_{i}$ for the position of $\boldsymbol{x}_{i}$ in the population. This method is customarily adopted to address problems with integer values. However it has been tested to produce reasonable values even in our case. The method is somewhat time consuming.

- Down Hill Simplex. This method has been developed by Nelder \& Mead (1965) and it has become the subject of many text books (Press et al. 1986). The action (13) is evaluated for some values or vertices of the coefficients $\boldsymbol{C}_{n, i}$ of the solutions (17) and minimized. Once the vertex coefficients have been determined, the method starts from the highest vertex, $\boldsymbol{x}_{h}$, and folds itself about the centroid, $\boldsymbol{x}_{c}$, of the remaining points to a new point, $\boldsymbol{x}_{a}$, in such a way that $\left\|\boldsymbol{x}_{a}-\boldsymbol{x}_{c}\right\| /\left\|\boldsymbol{x}_{c}-\boldsymbol{x}_{h}\right\|$ is equals to 1. If $\boldsymbol{x}_{a}$ falls in between the highest and lowest vertex, it replaces $\boldsymbol{x}_{h}$ and the process goes on. If $\boldsymbol{x}_{a}$ is the highest vertex, the method constructs $\boldsymbol{x}_{b}$ so that $\left\|\boldsymbol{x}_{c}-\boldsymbol{x}_{b}\right\| /\left\|\boldsymbol{x}_{c}-\boldsymbol{x}_{h}\right\|$ is equals to $1 / 2$. If $\boldsymbol{x}_{b}$ is lower than $\boldsymbol{x}_{h}$, the method replaces $\boldsymbol{x}_{h}$ with $\boldsymbol{x}_{b}$ and goes on, otherwise all vertex of the simplex are moved toward the lowest vertex $\boldsymbol{x}_{l}$ producing a new vertex $\boldsymbol{x}_{i}{ }_{i}$ so that $\left\|\boldsymbol{x}_{i}-\boldsymbol{x}^{\prime}{ }_{i}\right\| /\left\|\boldsymbol{x}_{i}-\boldsymbol{x}_{l}\right\|$ gets equal to $1 / 2$.

- Simulated Annealing. This is a random-walk search method. It generates a set of random starting points and for each one it takes a random direction along which to move on. If the new point yields a better solution it is accepted, otherwise the method calculates the probability $P$ and compares it to a random value $n \in[0,1]$. If $n<P$, the point is accepted even if the solution is not improved. The probability is given by $P=\exp \left[b\left(i, \Delta f, f_{0}\right)\right]$ where $b$ is defined by $b\left(i, \Delta f, f_{0}\right)=-\Delta f \log (i+1) / 10$, with $i$ the iteration number, $\Delta f$ the change in the target function, and $f_{0}$ thee value of the target function in the previous iteration (Press et al. 1986).

We have applied all the three methods using different random seeds and suitably changing the parameters of the method in use. This has required a large amount of computer time, however without leading to significant changes in the solutions for the velocity vectors of our galaxies. Indeed all velocity vectors differ from each other only by few $\mathrm{km} \mathrm{s}^{-1}$. The same for the action minimum.

Finally as we were more interested in the physical meaning of the result, we preferred not to look for the absolute minimum for the action. Instead, following Efron \& Tibshirani (1986) we considered the mean minimum value of the action resulting from a statistical interpretation of the results. As far as the orbits are concerned we performed many numerical experiments in which a fraction of galaxies of our sample has been randomly dropped from the configuration space and their distances randomly varied within the uncertainties, and finally the minimization has been recomputed. The final adopted values are for the statistical interpretation of the orbits. The results are presented in Table 6 .
Table 6. Mean value of the velocities derived from the analysis explained in the text.

\begin{tabular}{lrrr}
\hline \hline $\begin{array}{l}\text { Galaxy } \\
\text { name }\end{array}$ & $\begin{array}{r}v_{x}^{\mathrm{G}} \pm \sigma_{x} \\
\mathrm{~km} \mathrm{~s}^{-1}\end{array}$ & $\begin{array}{r}v_{y}^{\mathrm{G}} \pm \sigma_{y} \\
\mathrm{~km} \mathrm{~s}^{-1}\end{array}$ & $\begin{array}{r}v_{z}^{\mathrm{G}} \pm \sigma_{z} \\
\mathrm{~km} \mathrm{~s}^{-1}\end{array}$ \\
\hline IC 342 & $-298 \pm 10$ & $81 \pm 6$ & $-22 \pm 8$ \\
Maffei & $-353 \pm 15$ & $99 \pm 11$ & $-65 \pm 15$ \\
Andromeda & $-22 \pm 17$ & $-26 \pm 14$ & $2 \pm 18$ \\
MW & $9 \pm 9$ & $57 \pm 13$ & $-65 \pm 14$ \\
M 81 & $-380 \pm 16$ & $49 \pm 7$ & $96 \pm 11$ \\
Cen A & $722 \pm 22$ & $-210 \pm 19$ & $72 \pm 24$ \\
Sculptor & $820 \pm 12$ & $-49 \pm 18$ & $148 \pm 31$ \\
M 83 & $620 \pm 14$ & $-245 \pm 16$ & $87 \pm 6$ \\
\hline
\end{tabular}

The errors are relative quantities due to the statistical approach we have adopted.

A few simulations have been also calculated using the largest set of galaxies of Table 3 . All results are fully compatible with those of Table 6 . To fully and properly perform a statistical analysis one should also vary the velocities within their uncertainties. The estimated time cost of such analysis is very large. Fortunately, the small mass for most of galaxies in Table 3 ensures us that the final result would not significantly differ from the the present one.

\subsection{Analytical representation of the orbits}

As in the real space the tangent to the solution for the orbits given by Eq. (17) is expected to strongly depend on the number of terms in the polynomial expansion of the solution, we have extended it up to the ninth term $(N=9)$

$\boldsymbol{x}_{i}(a)=\boldsymbol{x}_{i}\left(t_{0}\right)+\sum_{n=0}^{8} \boldsymbol{C}_{i, n} f_{n}(a)$.

In the same way we have extended the analytical representation of Eq. (20) up to $N=4$ and $N=8$

$\boldsymbol{x}_{i}(t)=\boldsymbol{x}_{i, 0}+\sum_{n=1}^{8}\left(D(t)-D_{0}\right)^{n} \boldsymbol{C}_{n, i}$

To show the largest difference caused by the order of the analytical expansion of Eq. (32), in Table 7 we list the velocity vectors. As expected, the difference is rather small and comparable to that of Table 6.

Finally, we would like to remark that the analytical representation of the orbits and the minimization procedure do not affect the plane position which is only revealed by the Principal Direction analysis. Small errors in the plane determination could result from the approximation made in Eq. (28). Therefore, they are only due to the velocity section of the tangent bundle of the configuration space where our smooth Lagrangian function (13) is defined.

\subsection{Richness of the galaxy sample}

Finally, we evaluate the effect of changing the number of galaxies used to determine the force field acting on the LG. The analytical representation of the orbits is the one of Eq. (17) and the total galaxy sample is the sum of Tables 2 and 3 . The new velocity vectors are found to be very similar to those of Table 6 . This check is very time consuming and more subjected to the errors in 
Table 7. Local minimum velocity vectors for the solutions given by Eq. (32).

\begin{tabular}{cccc}
\hline \hline $\begin{array}{c}\text { Galaxy } \\
\text { name }\end{array}$ & $\begin{array}{c}v_{x}^{\mathrm{G}} \\
\mathrm{km} \mathrm{s}^{-1}\end{array}$ & $\begin{array}{c}v_{y}^{\mathrm{G}} \\
\mathrm{km} \mathrm{s}^{-1}\end{array}$ & $\begin{array}{c}v_{z}^{\mathrm{G}} \\
\mathrm{km} \mathrm{s}^{-1}\end{array}$ \\
\hline IC 342 & -295.807 & 79.9568 & -25.0462 \\
Maffei & -360.432 & 97.0 & -67.9 \\
Andromeda & -22.7 & -25.9 & 2.6 \\
MW & 7.2 & 57.2 & -66.3 \\
M 81 & -392.5 & 49.7 & 98.4 \\
Cen A & 727.9 & -200.6 & -5.3 \\
Sculptor & 824.5 & -51.7 & 150.6 \\
M 83 & 620.4 & -250.5 & 95.8 \\
\hline
\end{tabular}

These values are fully compatible with those listed in Table 6.

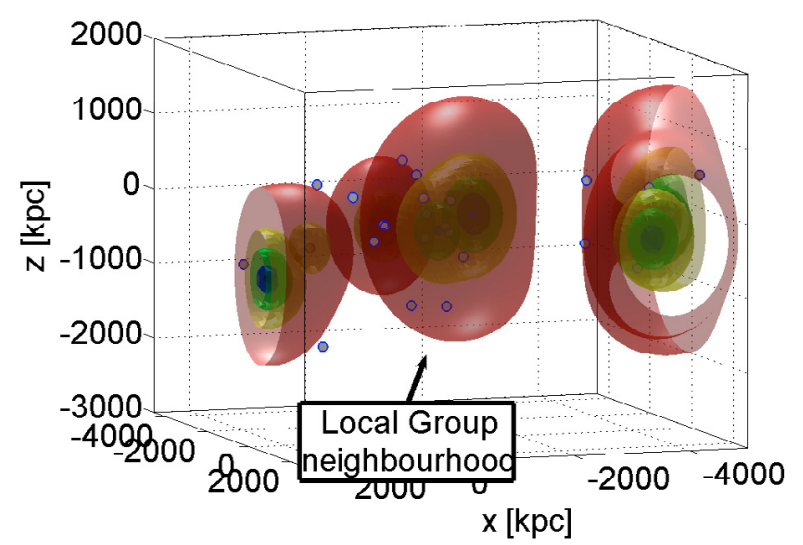

Fig. 11. Surfaces of constant gravitational potential for the galaxies and groups of galaxies given in Tables 3 and 2 . The colour code makes it evident how the spherical symmetry dominates. The position of the LG is indicated.

the distance values of Table 3 . Recalling that the number of interactions scales as $N_{p}^{2}$, the computational time is greatly increased. The little change in the velocity vectors that is found with the enlarged sample of galaxies is particularly interesting because it implies that the forces acting on the LG galaxies are primarily of internal origin. This is best illustrated by the shape of the equal gravitational potential surfaces and the field lines around each galaxy or galaxy group of the large sample (Tables 2 and 3) that are shown in Fig. 11. The gravitational potential around each object is nearly spherical and the field lines are nearly radial (see also Fig. 12 below). With these external gravitational fields, no significant effect can be produced on the LG galaxies even if the distance errors in some of the galaxies of 3 could be relevant.

\section{The external force field}

In this section we examine in more detail the spatial shape of the gravitational potential (and associate field lines) shown in Fig. 11 in order to better understand the effect of the external force field on the planar distribution of the LG galaxies discovered in Sect. 2. To this aim we limit ourselves to consider only the subgroup strictly belonging to the LG neighbourhood (see Table 2). As pointed out by Dunn \& Laflamme (1993) it is plausible that in the past dynamics and angular momentum (that ultimately depend on the quadrupole moment of the external gravitational potential, see Eq. (25)) of the LG have been strongly influenced not only by the two most massive spiral galaxies, M 31 and MW, but also by the nearest external galaxies. At the present time the dominant feature of the force field is

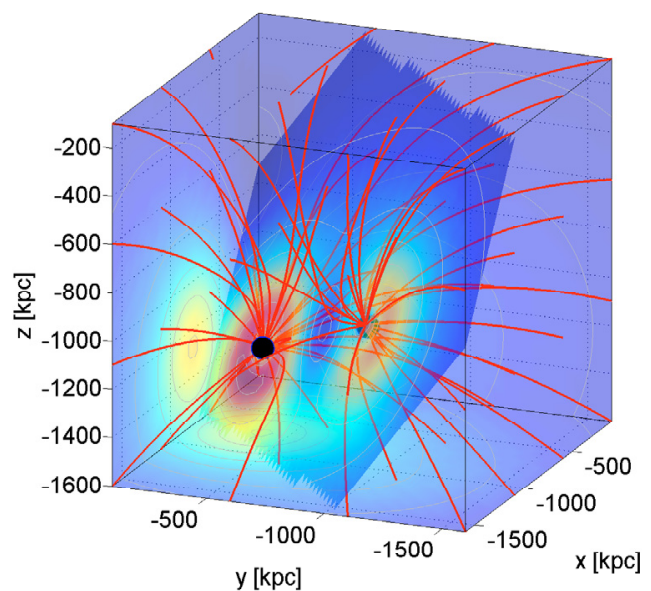

Fig. 12. The force field showing the radial behaviour of the streamlines of the MW and M 31 projected on the geometrical plane that minimizes the distances of the LG galaxies.

the two-body interaction between the two dominant spirals. The present-day situation is illustrated in Fig. 12 where we see the typical dipolar structure of the field line generated by MW and M 31. This ensures that only the central forces are governing the dynamics of the two major galaxies.

The plane corresponding to the central motion of MW and M 31 not necessarily has to coincide with the plane derived from geometrical arguments. Indeed the dynamics of the dwarf galaxies satellites of MW and M 31 could be fully incompatible with the planar distribution indicated by the geometry. The latter indeed could be a transient situation with no correlation with the orbital plane revealed by the minimum action. In Fig. 12 we plot the dipole force field at the present time $\left(t=t_{0}\right)$ over the geometrical plane. The dipole force field is the one derived from the minimum action (Sect. 5) whereas the geometrical plane is the one derived from Eq. (10). On the geometrical plane the presentday dipole structure of the force field is still evident.

To cast light on the dynamical significance of the geometrical plane, instead of investigating the dynamics due to the central force between the two major galaxies, we must clarify the influence of the external galaxies on both the geometrical plane and the central force plane.

Following Raychaudhury \& Lynden-Bell (1989), the force field minimizing the action can be expressed as

$\boldsymbol{F}(\boldsymbol{r})=G \sum_{\substack{j=1 \\ j \neq\{M W, M}}^{N_{p}} \frac{m_{j}\left(\boldsymbol{r}-\boldsymbol{r}_{j}\right)}{\left(\left\|\boldsymbol{r}-\boldsymbol{r}_{j}\right\|^{2}+\varepsilon^{2}\right)^{3 / 2}}$

Starting from this, we seek to understand how the external force field has influenced the past and present dynamics of the LG. This is the only way we can check the stability of the planar distribution by tightening together the static hint from the geometry and the dynamical hint from the orbits. The surprising result is shown in Fig. 13, which displays the face-on (top) and edgeon (bottom) projection of the geometrical plane, the lines of the force field and the position of the MW and M 31.

The force field of the external galaxies that minimize the action is clearly parallel to the plane. This means that the force perturbing the central dynamical forces of the $L G$ is dragging the plane toward the direction orthogonal to the normal direction, in other words the force field is pulling the plane along $i t$. The geometrical and central force planes coincide within the uncertainty of the procedure we adopted to bring all this into 


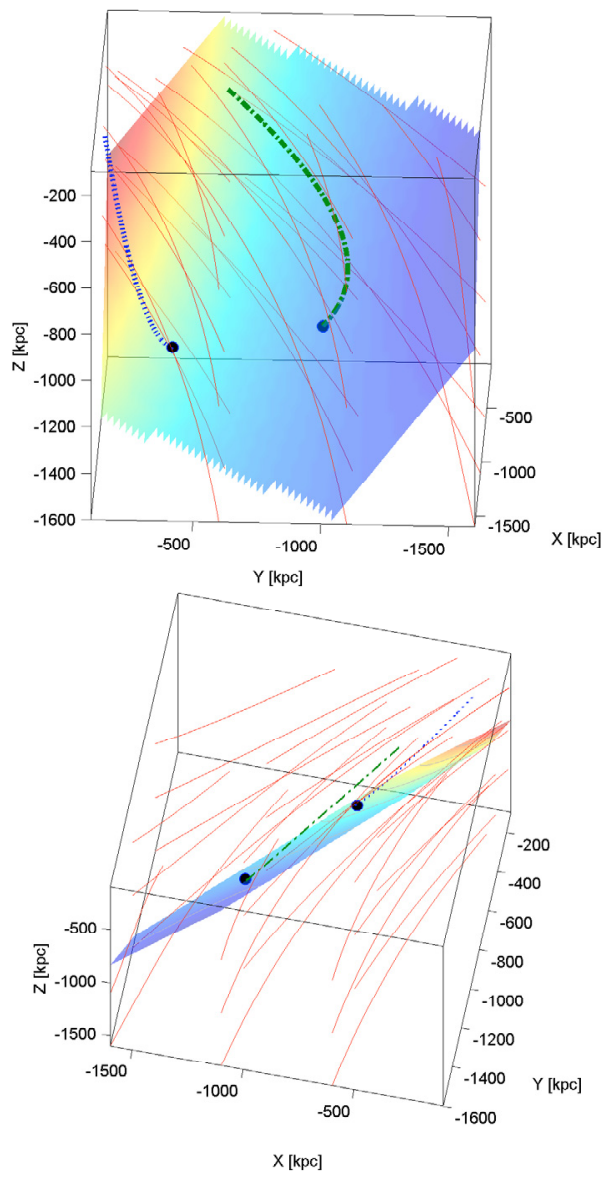

Fig. 13. Top panel: face-on view of the plane minimizing the action and the geometrical distances of the LG galaxies together with the field line of the gravitational interaction exerted by the external galaxies on the LG. Bottom panel: the same but viewed edge-on. Note how the field lines are nearly parallel to the plane. See the text for more details.

evidence. As we have not made any special assumption for the angular momentum of the LG this finding is very interesting. Indeed the solution with velocity vectors belonging to the geometrical plane does not give any hint about the inclination of the central force plane. An infinite set of different possibilities is compatible with the assumption that the velocity vectors of M 31 and MW belong to the geometrical plane, but the solution that minimize the action show that the orbits should be parallel to the geometrical plane, at least from $z=2$. Interestingly enough, the coincidence between the geometrical and central force planes stems from the minimum action. The external field might have been significantly different at epochs earlier than $z=2$ (Dunn \& Laflamme 1993) but ever since it did not change too much. The orbits derived from the minimum action (Sect. 5) belong to the geometrical plane. The two planes have been coincident for a large fraction of the Hubble time (nearly all).

This makes it possible to constrain the proper motions of the dwarf galaxies external to the LG (Sawa \& Fujimoto 2005), or more realistically to constrain the dwarf galaxies (mostly irregulars) of the LG that are preferentially located at large distances from the two major galaxies and are subjected to a lower degree of spherical symmetry in the force field due to the dark matter halo of the two massive spirals, see (Pasetto et al. 2003). The force field lines shown in Fig. 13 are likely to describe, with no significant variations with time, the direction and intensity of the force since very early epochs, say $z \cong 2$.

\section{Conclusions}

In this paper we have investigated the spatial distribution of the galaxies in the LG. They seem to share a common plane. The plane has been first geometrically singled out and then dynamically interpreted.

The geometrical discovery has been made using the Principal Component Analysis for an un-weighed sample. The plane we find does not coincide with those recently suggested by Sawa $\&$ Fujimoto (2005), on the base of the sky-projected distribution, and Kunkel (1979) and Grebel et al. (1999) from dynamical studies limited to the MW and M 31 sub-systems.

Major uncertainty of our analysis is the issue of the mass tracers. Indeed the luminous objects have been used to derive from merely geometrical arguments the plane minimizing their distances to this. We have reasonably supposed that pure Dark Matter haloes do not likely exist in the LG at least for redshifts $z \leq 2$. However, the presence of such pure Dark Matter haloes, while not affecting the determination of the geometrical plane, could affect on the orbital motions and the planar distribution of galaxies that we have derived from the dynamical analysis.

The second part of the article is devoted to investigate the dynamics of the two dominant galaxies of the LG, MW and M 31, under the action of external forces exerted by nearby galaxies or galaxy groups external to the LG. The aim is to prove whether the planar distribution we have found is stable in time or it is a mere coincidence that will disappear in the future. We have demonstrated that the force field acting on the dynamics of the LG as a whole is compatible with the geometrical plane, in other words with a planar distribution that remain stable for very long periods of time. The result is that there is no external force field able to destroy the planar distribution. Once again the possible presence of undetected pure Dark Matter haloes may be a drawback of the present study.

Putting together the information from the geometrical and dynamical analysis the real thickness of the plane is estimated to be about $200 \mathrm{kpc}$.

Furthermore, despite the uncertainties, there is a strong hint that the motion of the galaxies inside the LG is due to a central force field. The long membership of the two spiral galaxies to the plane, for about $10 \mathrm{Gyr}$, provides some information on the virialization state of the LG and the proper motions. However, despite the extensive use by Sawa \& Fujimoto (2005), the presence of a plane should be adopted only to determine of proper motions of the dwarf galaxies in the outskirts of the LG, as close to the dominant spirals the gravitational potential and the fly-by encounters are far from being collisionless and scatter phenomena can complicate the description (see Pasetto \& Chiosi 2006, in preparation). We would like to remark that from the results of Table 6 we can derive the proper motions expected for M 31. Following Vallenari et al. (2006) (their Eq. (4)) and assuming that the Sun is at rest in the Local Standard of Rest system, the velocity system $(U, V, W)$ of Eq. (4) in Vallenari et al. (2006) is aligned with our velocity system. Therefore the peculiar velocity of M 31 is simply the velocity given in Table 6 once translated into the velocity system centered on the MW. We obtain $\left(\mu_{l}, \mu_{b}\right)=\left(-3.03 \times 10^{-4},-3.92 \times 10^{-4}\right)$ arcsec $\mathrm{yr}^{-1}$, fully compatible with Dunn \& Laflamme (1993). As expected the proper motions turn out to be small due to the nearly parallel motion of M 31 with respect to the MW. This result also indirectly confirms that the numerical errors in our analysis are quite small as we derive a radial velocity of $\mathrm{M} 31$ equal to $-115 \mathrm{~km} \mathrm{~s}^{-1}$ which is virtually identical to the one we have initially imposed, i.e. the observational value $v_{r}=-113 \mathrm{~km} \mathrm{~s}^{-1}$. 


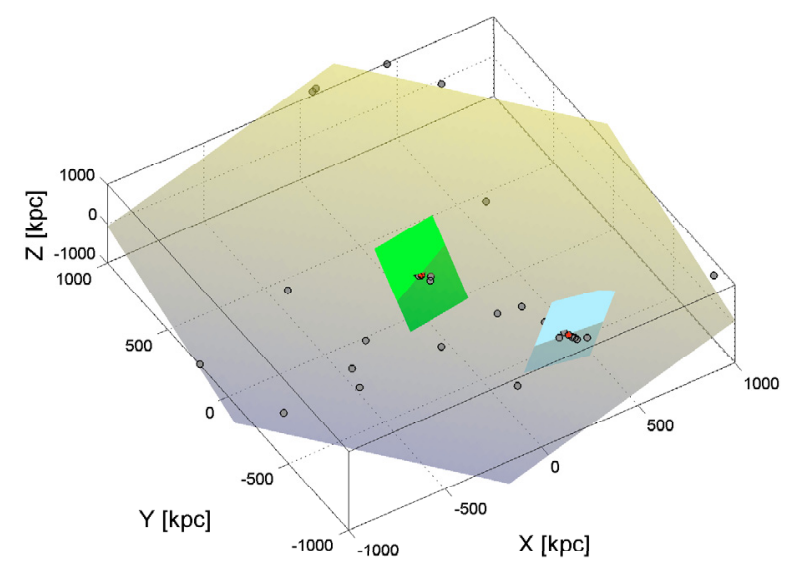

Fig. 14. The planes for the dwarf galaxies around the MW (Kroupa et al. 2005) and M 31 (Koch \& Grebel 2006) compared to our plane. Unfortunately, we cannot show the plane of Sawa \& Fujimoto (2005) as no equation for it has been given explicitly by the authors.

Finally in Fig. 14 we plot and compare with ours the planes found by Kroupa et al. (2005) around the MW and by Koch \& Grebel (2006) around M 31. First of all, the planes found by Kroupa et al. (2005) and Koch \& Grebel (2006) differ from each other (have different normals). While the normal to the plane around MW seems to be aligned with the direction toward M 31 this is not the case for the plane around M 31 whose normal does not align with the direction towards the MW. Second, both planes do not coincide with our plane. The problem is rather complicated and in part mirrors the different methods that have been adopted in the three studies to address the subject and to determine the planes. They stem indeed from different dynamical considerations. In brief, the gravitational interaction among galaxies can be studied in two regimes. On long distances it can be investigated as a collisionless process best described by a statistical method. This is the approach of the present study as well as of many others in literature. Basing on this, we have derived the spatial structure and evolution of the MW and M 31 complexes together with their satellites. On short distances, the gravitational interaction is best modelled by collisional dynamics. This is the typical case of studies on the orbits of satellites of big mother galaxies and the effect of these latter on the spatial distribution of their nearby dwarf galaxies. In this context Koch \& Grebel (2006) suggested for the M 31 group a tidal break up of a pre-existing dominant galaxy. This fact could partially explain the lack of collinearity between the planes for the M 31 and MW complexes. However, it is likely that also the progenitor of M 31 has been influenced by the external force field we have analysed in the present study. Furthermore, the seemingly parallel pull of the external field minimizing the action could have influenced, roughly in the same manner, both the proto-M 31 and the proto-MW (if any). As a final consideration on this issue, it may well be that on a local scale, i.e. when studying the dynamical behaviour of the satellite galaxies belonging to the MW and M 31 complexes, they seem to lie on planes with different inclination, but the generalisation of this result to the whole LG may not be correct. On the grand scale of the whole LG all galaxies seem to belong to a rather thin slab with its own (different) inclination which is dot destroyed by external actions. A study of the LG including both the collisional effects among interacting dwarf galaxies and the tidal effects by the external field on the orbits of the dwarf galaxies is underway (Pasetto \& Chiosi 2006, in preparation).
In this paper we tried to set the ground for future analysis in which the two regimes for the gravitational interaction can be taken into account. Specifically, we tried to impose constraints to the tangent bundle space of a smooth collisionless Lagrangian function generated by a group of galaxy clusters, using equations that stem from a local analysis and the probable existence of a plane revealed by merely geometrical considerations. The Lagrangian is constrained only at the time-boundaries of its region by means of the velocity space over there. In other words, the action minimization is constrained at the two temporal extremes: at the origin of the Universe by means of the Hamilton Principle (in the Peebles interpretation) and at the present time by means of the observational velocities and positions. Therefore, the action minimization here is one of the first order, i.e. a free, unconstrained minimization but for the temporal extremes with no other constraint along the minimization path. The next step forward would be to include the collisional regime. Unfortunately, in this case the dynamical interactions easily loose memory of the initial conditions. Therefore the dynamics of the LG must be investigated by imposing additional new and suitable constraints on the tangent bundle space of the Lagrangian. Precious hints come from the stellar content of the mother and dwarf galaxies of the LG Koch \& Grebel (2006). In other words, the dynamical history of individual galaxies is recorded not only in their space-time geodesics or their past orbits (that anyhow are still poorly determined despite the many efforts in literature) but also in their past history of star formation and chemical enrichment on which we know lots more, see e.g. the reconstruction of this information made over the past two decades deciphering the colour-magnitude diagrams of their stellar populations (see Gallart et al. 2005, for a recent review of the subject and references therein). The target is ambitious but feasible.

Acknowledgements. This study was financed by the University of Padua by means of a post-doc fellowship to S. Pasetto and the Department of Astronomy by providing indirect logistic support (computers, infrastructure, etc). No financial resource has been allocated to the project by the Italian Ministry of Education, University and Research (MIUR).

\section{References}

Barnard, E. E. 1917, AJ, 30, 175

Bennett, C. L., Halpern, M., Hinshaw, G., et al. 2003, ApJS, 148, 1

Bondi, H. 1960, The universe at large (Garden City, N.Y.: Anchor Books), 1st ed. Branchini, E., Eldar, A., \& Nusser, A. 2002, MNRAS, 335, 53

Brenier, Y., Frisch, U., Hénon, M., et al. 2003, MNRAS, 346, 501

Buta, R. J., \& McCall, M. L. 1983, MNRAS, 205, 131

Byrd, G., Valtonen, M., McCall, M., \& Innanen, K. 1994, AJ, 107, 2055

Carrasco, E. R., Mendes de Oliveira, C., \& Infante, L. 2004, in Clusters of

Galaxies: Probes of Cosmological Structure and Galaxy Evolution, ed. J. S. Mulchaey, A. Dressler, \& A. Oemler

Coles, P., \& Lucchin, F. 2002, Cosmology: The Origin and Evolution of Cosmic Structure, Second Edition (Wiley-VCH)

Courteau, S., \& van den Bergh, S. 1999, AJ, 118, 337

Davis, M., \& Peebles, P. J. E. 1983, ApJ, 267, 465

De Marco, G. 1994, ANALISI DUE/2 (Zanichelli, DECIBEL)

Diemand, J. 2005, in Near-fields cosmology with dwarf elliptical galaxies, ed. H. Jerjen, \& B. Binggeli, IAU Colloq., 198, 211

Diemand, J., Moore, B., \& Stadel, J. 2005, Nature, 433, 389

Dunn, A. M., \& Laflamme, R. 1993, MNRAS, 264, 865

Dunn, A. M., \& Laflamme, R. 1995, ApJ, 443, L1

Efron, B., \& Tibshirani, R. 1986, Statistical Sci., 1, 54

Einasto, J., \& Lynden-Bell, D. 1982, MNRAS, 199, 67

Evans, N. W., \& Wilkinson, M. I. 2000, MNRAS, 316, 929

Evans, N. W., Wilkinson, M. I., Guhathakurta, P., Grebel, E. K., \& Vogt, S. S. 2000, ApJ, 540, L9

Fingerhut, R. L., McCall, M. L., De Robertis, M., et al. 2003, ApJ, 587, 672 
Fleiner, C. 1997, Parallel Optimizations: Advanced Constructs and Compiler Optimizations for a Parallel, Object Oriented, Shared Memory Language running on a distributed System, Tech. Rep. TR-97-014, Berkeley, CA

Fusi Pecci, F., Bellazzini, M., Cacciari, C., \& Ferraro, F. R. 1995, AJ, 110, 1664

Gallart, C., Zoccali, M., \& Aparicio, A. 2005, ARA\&A, 43, 387

Giavalisco, M., Mancinelli, B., Mancinelli, P. J., \& Yahil, A. 1993, ApJ, 411, 9

Goldberg, D. M. 2001a, ApJ, 552, 413

Goldberg, D. M. 2001b, ApJ, 550, 87

Goldberg, D. M., \& Spergel, D. N. 2000, ApJ, 544, 21

Gott, J. R., \& Thuan, T. X. 1978, ApJ, 223, 426

Governato, F., Moore, B., Cen, R., et al. 1997, New Astron., 2, 91

Grebel, E. K. 2001, in Microlensing 2000: A New Era of Microlensing Astrophysics, ed. J. W. Menzies, \& P. D. Sackett, ASP Conf. Ser., 239, 280

Grebel, E. K., Kolatt, T., \& Brandner, W. 1999, in IAU Symp., ed. P. Whitelock, \& R. Cannon, 447

Gunn, J. E., \& Gott, J. R. I. 1972, ApJ, 176, 1

Hartwick, F. D. A. 2000, AJ, 119, 2248

Heath, D. J. 1977, MNRAS, 179, 351

Hoyle, F. 1951, in Problems of Cosmical Aerodynamics; Proceedings of a Symposium on the Motion of Gaseous Masses of Cosmical Dimensions held at Paris, August 16-19, 1949, UNESCO meeting jointly sponsored by IAU and IUTAM, 195

Jobson, J. 1994, Applied Multivariate Data Analysis, Springer Texts in Statistics

Kahn, F. D., \& Woltjer, L. 1959, ApJ, 130, 705

Kang, X., Mao, S., Gao, L., \& Jing, Y. P. 2005, A\&A, 437, 383

Karachentsev, I. D. 2005, AJ, 129, 178

Karachentsev, I. D., Dolphin, A. E., Geisler, D., et al. 2002, A\&A, 383, 125

Klypin, A., Gottlöber, S., Kravtsov, A. V., \& Khokhlov, A. M. 1999, ApJ, 516, 530

Knebe, A., Gill, S. P. D., Gibson, B. K., et al. 2004, ApJ, 603, 7

Koch, A., \& Grebel, E. K. 2006, AJ, 131, 1405

Kroupa, P., Theis, C., \& Boily, C. M. 2005, A\&A, 431, 517

Kunkel, W. E. 1979, ApJ, 228, 718

Libeskind, N. I., Frenk, C. S., Cole, S., et al. 2005, MNRAS, 363, 146

Lynden-Bell, D. 1981, The Observatory, 101, 111

Lynden-Bell, D., \& Lin, D. N. C. 1977, MNRAS, 181, 37

Mateo, M. L. 1998, ARA\&A, 36, 435

McCall, M. L. 1989, AJ, 97, 1341

Mishra, R. 1985, MNRAS, 212, 163

Moore, B., Ghigna, S., Governato, F., et al. 1999, ApJ, 524, L19

Mould, J., Ferrarese, L., Stetson, P. B., Tonry, J., \& Blakeslee, J. 2005, AAS, 207

Natarajan, P., \& Springel, V. 2004, ApJ, 617, L13

Nelder, J. A., \& Mead, R. 1965, 8, 308

Nusser, A., \& Branchini, E. 2000, MNRAS, 313, 587

Ostriker, J. P., \& Peebles, P. J. E. 1973, ApJ, 186, 467

Ostriker, J. P., Peebles, P. J. E., \& Yahil, A. 1974, ApJ, 193, L1

Pasetto, S., Chiosi, C., \& Carraro, G. 2003, A\&A, 405, 931
Peebles, P. J. E. 1969, ApJ, 155, 393

Peebles, P. J. E. 1974, ApJ, 189, L51

Peebles, P. J. E. 1980, The large-scale structure of the universe (Princeton, N.J.,

USA: Princeton University Press)

Peebles, P. J. E. 1989, ApJ, 344, L53

Peebles, P. J. E. 1990, ApJ, 362, 1

Peebles, P. J. E. 1994, ApJ, 429, 43

Peebles, P. J. E. 1995, ApJ, 449, 52

Peebles, P. J. E., Melott, A. L., Holmes, M. R., \& Jiang, L. R. 1989, ApJ, 345, 108

Peebles, P. J. E., Phelps, S. D., Shaya, E. J., \& Tully, R. B. 2001, ApJ, 554, 104

Phelps, S. D. 2002, ApJ, 575, 1

Press, W. H., Flannery, B. P., \& Teukolsky, S. A. 1986, Numerical recipes. The art of scientific computing (Cambridge: University Press, 1986)

Raychaudhury, S., \& Lynden-Bell, D. 1989, MNRAS, 240, 195

Saha, A., Claver, J., \& Hoessel, J. G. 2002, AJ, 124, 839

Saha, R., Jain, P., \& Souradeep, T. 2006, ApJ, 645, L89

Sales, L., \& Lambas, D. G. 2004, MNRAS, 348, 1236

Saletan, E. J., \& Cromer, A. H. 1970, Am. J. Phys, 38, 892

Sawa, T., \& Fujimoto, M. 2005, PASJ, 57, 429

Schmoldt, I. M., \& Saha, P. 1998, AJ, 115, 2231

Sharpe, J., Rowan-Robinson, M., Canavezes, A., et al. 1999, in Observational Cosmology: The Development of Galaxy Systems, ed. G. Giuricin, M. Mezzetti, \& P. Salucci, ASP Conf. Ser., 176, 280

Shaya, E. J., Peebles, P. J. E., \& Tully, R. B. 1995, ApJ, 454, 15

Storn, R., \& Price, K. 1995, Differential Evolution - a simple and efficient adaptive scheme for global optimization over continuous spaces, Tech. Rep. TR95-012, Berkeley, CA

Thim, F., Tammann, G. A., Saha, A., et al. 2003, ApJ, 590, 256

Thuan, T. X., \& Gott, J. R. 1977, ApJ, 216, 194

Tully, R. B. 1988, Nearby galaxies catalog (Cambridge and New York: Cambridge University Press), 221

Tully, R. B., \& Fisher, J. R. 1988, Annales de Geophysique

Vallenari, A., Pasetto, S., Bertelli, G., et al. 2006, A\&A, 451, 125

Valtonen, M. J., Byrd, G. G., McCall, M. L., \& Innanen, K. A. 1993, AJ, 105, 886

van den Bergh, S. 1999, A\&A Rev., 9, 273

Walker, A. R. 2003, Stellar Candles for the Extragalactic Distance Scale, LNP, 635,265

White, S. D. M., Tully, R. B., \& Davis, M. 1988, ApJ, 333, L45

Whiting, A. B. 2000, ApJ, 533, 50

Whittaker, E. T. 1989, A Treatise on the Analytical Dynamics of Particles and Rigid Bodies (Cambridge, UK: Cambridge University Press)

Yahil, A., Tammann, G. A., \& Sandage, A. 1977, ApJ, 217, 903

Zentner, A. R., Kravtsov, A. V., Gnedin, O. Y., \& Klypin, A. A. 2005, ApJ, 629, 219 
S. Pasetto and C. Chiosi: Planar distribution of galaxies in the Local Group, Online Material $p 1$

\section{Online Material}




\section{Appendix A}

Here we describe the simple method used to minimize the distance expressed by Eq. (3) subjected to the constraint given in Eq. (4) and the constraint that the plane we are looking for must be orthogonal the vector joining M 31 and MW, i.e. $\boldsymbol{a}=\left(a_{x}, a_{y}, a_{z}\right)$. This additional holonomic constraint can be written as $\boldsymbol{n} \cdot \boldsymbol{a}=0$. The problem can be further simplified assuming as origin of the coordinate system of reference one of the two major galaxies. This means that the constant $c$ of Eq. (2) can be set to zero $c=0$. Therefore $\boldsymbol{n} \cdot \boldsymbol{a}=0$ and $|\boldsymbol{n}|^{2}-1=0$ are the system of independent constraints. The distance defined by Eq. (3) in the Euclidean space $E^{3}$ will present a stationary point $\boldsymbol{n}$ if the gradients of the constraints in $\boldsymbol{n}$ are able to express $\nabla D$ as a linear combination of them, i.e are a basis for $N_{n} E^{3}$, the space orthogonal to the manifold of $\boldsymbol{n}$. This leads to

$2 \sum_{g=1}^{N_{p}}\left\langle\boldsymbol{n}, \boldsymbol{x}^{(g)}\right\rangle \boldsymbol{x}^{(g)}=2 \lambda_{1} \boldsymbol{n}+\lambda_{2} \boldsymbol{a}$.

For simplicity we express the position as

$\boldsymbol{A}\left(=A_{i j}\right) \equiv 2 \sum_{g=1}^{N}\left(x_{i} x_{j}\right)^{(g)}$

and shortly rewrite the previous equation as

$\langle\boldsymbol{A}, \boldsymbol{n}\rangle=\lambda_{1} \boldsymbol{n}+\lambda_{2} \boldsymbol{a}$.

This is a non homogeneous system of linear equations with parameters $\lambda_{1}$ and $\lambda_{2}$. We can also write

$\langle\boldsymbol{A}, \boldsymbol{n}\rangle-\lambda_{1} \boldsymbol{n}=\lambda_{2} \boldsymbol{a}$

and re-cast the system as

$\left(\boldsymbol{A}-\lambda_{1} \boldsymbol{I}\right) \boldsymbol{n}=\lambda_{2} \boldsymbol{a}$

The rank of the symmetric matrix in $\mathfrak{R}^{3}$ is 3 , so that $\operatorname{det}\left[\boldsymbol{A}-\lambda_{1} \boldsymbol{I}\right] \neq 0$ which means that $\lambda_{1}$ is not an eigen-value for $\boldsymbol{A}$. In this case a solution of the system A.3 surely exists because the rank of the matrix $\boldsymbol{M} \equiv\left(\boldsymbol{A}-\lambda_{1} \boldsymbol{I}\right)$ is the same as the rank of the same matrix completed with the column $\left\{\lambda_{2} \boldsymbol{a}\right\}$ : $\left\{\boldsymbol{M}, \lambda_{2} \boldsymbol{a}\right\}$. This can be translated into a condition on the parameter $\lambda_{1}$, not on $\lambda_{2}$. The solutions form a vectorial space and they can be determined with the Kramer's method.
However, as we are more interested in the components of the ortho-normal vector $\boldsymbol{n}$ more than in the Lagrange's multipliers $\lambda_{1}$ and $\lambda_{2}$, we can work directly on the system (A.3). Taking the scalar product of both members of (A.3) we obtain

$$
\begin{aligned}
& \langle\langle\boldsymbol{A}, \boldsymbol{n}\rangle, \boldsymbol{n}\rangle=\left\langle\lambda_{1} \boldsymbol{n}, \boldsymbol{n}\right\rangle+\left\langle\lambda_{2} \boldsymbol{a}, \boldsymbol{n}\right\rangle \\
& \langle\boldsymbol{A} \boldsymbol{n}, \boldsymbol{n}\rangle=\lambda_{1}\langle\boldsymbol{n}, \boldsymbol{n}\rangle+\boldsymbol{n}\langle\boldsymbol{a}, \boldsymbol{n}\rangle \\
& \langle\boldsymbol{A} \boldsymbol{n}, \boldsymbol{n}\rangle=\lambda_{1},
\end{aligned}
$$

where once again we find the dependence of the solution on $\lambda_{1}$. We can avoid all this by explicitly writing the components as

$n_{j}\left[\sum_{g=1}^{N}\left(x_{j}^{g} \sum_{i=1}^{3} n_{i} x_{i}^{g}\right)\right]=\lambda_{1} j=1,2,3 \Leftrightarrow$

$\left\{\begin{aligned} n_{1}\left[\sum_{g=1}^{N}\left(x_{1}^{g} \sum_{i=1}^{3} n_{i} x_{i}^{g}\right)\right] & =\lambda_{1} \\ n_{2}\left[\sum_{g=1}^{N}\left(x_{2}^{g} \sum_{i=1}^{3} n_{i} x_{i}^{g}\right)\right] & =\lambda_{1} \\ n_{3}\left[\sum_{g=1}^{N}\left(x_{3}^{g} \sum_{i=1}^{3} n_{i} x_{i}^{g}\right)\right] & =\lambda_{1},\end{aligned}\right.$

which means that

$\left\{\begin{array}{l}n_{1}\left[\sum_{g=1}^{N}\left(x_{1}^{g} \sum_{i=1}^{3} n_{i} x_{i}^{g}\right)\right] \\ n_{1}\left[\sum_{g=1}^{N}\left(x_{1}^{g} \sum_{i=1}^{3} n_{i} x_{i}^{g}\right)\right] \\ n_{2}\left[\sum_{g=1}^{N}\left(x_{2}^{g} \sum_{i=1}^{3} n_{i} x_{i}^{g}\right)\right]=n_{3}\left[\sum_{g=1}^{N}\left(x_{2}^{g} \sum_{i=1}^{3} n_{i} x_{i}^{g} x_{i}^{g} \sum_{i=1}^{3} n_{i} x_{i}^{g}\right)\right] \\ \left.=\sum_{g=1}^{N}\left(x_{3}^{g} \sum_{i=1}^{3} n_{i} x_{i}^{g}\right)\right] .\end{array}\right.$

This is a system whose solutions $\left\{n_{1}, n_{2}, n_{3}\right\}$ yield the vector we are looking for. The numerical solution is straightforward. 